\title{
Über die Einteilchensingularitäten in Relativistischen Quantenfeldtheorien
}

\author{
A. H. VöLKEL \\ IT. Institut für Theoretische Physik - Universität Hamburg
}

Eingegangen am 10. Dezember 1965

\begin{abstract}
By subtraction of products of three-point functions the four-point functions in relativistic quantum field theory are decomposed into two parts, in one of which there does not occur any mass-shell-singularity in the variables $k_{\alpha}+k_{\beta}(\alpha \neq \beta ; 1 \leqq \alpha, \beta \leqq 4)$. All these singularities are given explicitly by the kernels of the products of the three-point functions. - Necessary and sufficient conditions for the non-triviality of unitary $S$-matrices or some of their elements are proved in terms of statements on the occurence of mass-shell-singularities in the vacuum expectation values of field operators. The strongest result we have gained is: If $\delta\left(\left(\sum_{j=2}^{N-2} k_{j}\right)^{2}-m^{2}\right)\left[\left(\sum_{j=2}^{N-2} k_{j}\right)^{2}-m^{2}\right] \cdot\left\langle 0\left|\underset{j=1}{N} \tilde{\mathrm{A}}\left(k_{j}\right)\right| 0\right\rangle$ is equal to zero for some $N>3$, then all transition amplitudes $T_{2 \rightarrow n}$ vanish for every $n$.
\end{abstract}

\section{Problemstellung}

Wir betrachten eine skalare Feldtheorie, die durch eine operatorwertige Distribution $\mathrm{A}[f]=\int \mathrm{A}(x) f(x) d x$ in einem Hilbertraum $\mathfrak{S}$ beschrieben wird. Die Operatoren $A[f]$ sind unbeschränkt mit einem in F) dichten Definitionsbereich $D[\mathrm{~A}] . f(x)$ soll ein Element aus einem geeigneten Testfunktionenraum, z. B. $\mathfrak{S}_{4 n}$ sein; (d. h. $f(x)$ ist eine $C^{\infty}$-Funktion über dem reellen Minkowski-Raum, die im Unendlichen stärker als jede reziproke Potenz abfällt). Wir werden im folgenden an Stelle der Distribution A [f] immer den Begriff der ,,verallgemeinerten Funktion $\mathrm{A}(x)^{\varsigma}$ benutzen. Alle über dem reellen Minkowski-Raum auftretenden Funktionen sind dabei als Distributionen über $\mathfrak{S}_{4 n}$ aufzufassen [1], [2], [3], [4].

Das Feld A $(x)$ soll die folgenden Eigenschaften haben [4], [5], [8]:

(AI) Invarianz unter der Poincaré-Gruppe iLg ${ }_{+}^{\top}$

(AII) Spektrumbedingung

(AIII) Lokalität

(AIV) Vollständigkeit

Bezeichnen wir die Bereiche $p^{2}>b^{2} \pm p^{(0)}>0$ des MinkowskiRaumes mit $V_{ \pm}^{b}$ und ihren Abschluß mit $\overline{V_{ \pm}^{b}}$, so bedeutet die Spektrumsbedingung, daß die Spektralzerlegung des Translationsoperators $T(a)$

$$
\mathrm{T}(a)=\int e^{i p a} d \mathrm{E}(p) \quad p a=p^{(0)} a^{(0)}-\mathbf{p a}
$$


die folgenden Eigenschaften hat:

$$
\begin{array}{r}
d \mathrm{E}(p)=\theta(p)\left\{\delta(p)|0\rangle\left\langle 0\left|+\delta\left(p^{2}-m^{2}\right)\right| p, m\right\rangle\langle m, p|\right\} d^{4} p+d \varrho(p) \\
\text { Träger } d \varrho(p) \subseteq \overline{V_{+}^{2 m}}
\end{array}
$$

Dabei ist $|0\rangle$ der eindeutige Vakuumzustand und $|p, m\rangle$ ein Einteilchenzustand zur Masse $m$ und zum Impuls $p$.

Nach Wightman [4], [5] wird eine solche Theorie vollständig durch die Angabe einer Folge von Funktionen $W_{n}\left(x_{1}, x_{2}, \ldots, x_{n}\right)(n=1,2, \ldots)$ beschrieben, die mit den Vakuumerwartungswerten von $n$-fachen Produkten der Feldoperatoren $\mathrm{A}(x)$ übereinstimmen.

$$
\begin{gathered}
W_{n}\left(x_{1}, \ldots, x_{n}\right)=<0\left|\mathrm{~A}\left(x_{1}\right) \ldots \mathrm{A}\left(x_{n}\right)\right| 0> \\
\widetilde{W}_{n}\left(k_{1}, \ldots, k_{n}\right)=\frac{1}{(2 \pi)^{\frac{5}{2} n}} \int d x_{1} d x_{2} \ldots d x_{n} e^{i k x} W_{n}\left(x_{1}, \ldots, x_{n}\right) .
\end{gathered}
$$

Die Wightman-Funktionen haben die folgenden zu (AI)-(AIV) äquivalenten Eigenschaften:

(WI) Lorentzinvarianz:

$W_{n}\left(\Lambda x_{1}+a, \ldots, \Lambda x_{n}+a\right)=W_{n}\left(x_{1}, \ldots, x_{n}\right)$ für $(\Lambda, a) \in i L g_{+}$

(WII) Spektrumsbedingung):

Träger $\widetilde{W}_{n}\left(k_{1}, \ldots, k_{n}\right) \leqq M$ mit

$$
\begin{gathered}
M=\left\{k: \sum_{j=1}^{r} k_{j} \in \frac{\circ}{V_{+}^{2 m}} \cup\{0\} \text { für } r=1 ; \ldots ; n-1 \text { und } \sum_{j=1}^{n} k_{j}=0\right\} \\
\frac{\circ}{V_{ \pm}^{2 m}}=: \overline{V_{ \pm}^{2 m}} \cup\left\{k: k^{2}-m^{2}=0 ; \pm k^{(0)}>0\right\} \\
W_{2}(x ; y)=i \Delta_{+}^{m}(x-y)+i \int_{4 m_{2}}^{\infty} d \varrho(\mu) \Delta_{+}^{\mu}(x-y)
\end{gathered}
$$

(WIII) Lokalität:

$W\left(x_{1}, \ldots, x_{j}, x_{j+1}, \ldots, x_{n}\right)-W\left(x_{1}, \ldots, x_{j+1}, x_{j}, \ldots, x_{n}\right)=0$

für $\left(x_{j}-x_{j+1}\right)^{2}<0$.

$\mathrm{Zu}$ diesen linearen Bedingungen kommt noch eine weitere nichtlineare hinzu, die sich unmittelbar aus der Positivität der Norm in $\mathfrak{S}$ ergibt. Diese nichtlineare Bedingung spielt bei den weiteren Betrachtungen keine Rolle, so daß auf ihre Angabe verzichtet werden kann.

Infolge der Spektrumsbedingungen sind die Wightman-Funktionen im Ortsraum Randwerte von analytischen Funktionen mit gewissen asymptotischen Beschränktheitsbedingungen [5].

Die relativistische Kovarianz führt zu einer Vergrößerung dieses Regularitätsgebietes [6], [7], [8]. Schließlich ergibt dann die Lokalität, daß alle permutierten $W$-Funktionen analytische Fortsetzungen voneinander sind; d. h. die Wightman-Funktionen sind Randwerte einer 
einzigen in einem einfach zusammenhängenden Bereich eindeutigen und analytischen Funktion [8], [9], [10]. (Vergleiche die analogen Eigenschaften der retardierten Funktionen im Impulsraum: Abschnitt II.)

Fügt man in den Vakuumserwartungswerten (3) zwischen zwei Feldoperatoren ein System von Zwischenzuständen ein, so führt jeder dieser Zustände zu bestimmten Arten von Singularitäten im Impulsraum. Wie aus der Spektralzerlegung des Translationsoperators (1), (2) ersichtlich ist, liefern z. B. die Vakuumzustände $\delta$-Funktionen $\delta\left(\sum p_{i}\right)$, und ebenso die Einteilchenzustände $\delta\left(\left(\sum p_{i}\right)^{2}-m^{2}\right)$.

Hinzu kommen im allgemeinen auf der Massenschale noch Singularitäten der Art $\left[\left(\sum p_{i}\right)^{2}-m^{2}\right]^{-1}$ (siehe Abschnitt IV).

Da andererseits diese Singularitäten das asymptotische Verhalten der Wightman-Funktionen für große Separationen der Argumente im Ortsraum bestimmen, ist es für die Untersuchung aller mit der asymptotischen Teilcheninterpretation, der S-Matrix und ähnlichen Problemen zusammenhängenden Fragen wichtig, diese Singularitäten explizite aus den Wightman-Funktionen herauszupräparieren, und zwar möglichst so, daß die bei den Singularitäten aufgetretenen Koeffizienten wiederum Wightman-Funktionen bzw. „Produkte“ von Wightman-Funktionen mit einer geringeren Anzahl von Argumenten sind.

Im Falle der Vakuumsingularitäten ist dies vollständig durch die folgende suczessive Definition der sog. trunkierten Wightman-Funktionen $W_{n}^{T}\left(x_{1 i}, \ldots, x_{n}\right)$ gelungen [11], [12]

$$
\begin{aligned}
W^{T}\left(x_{1}, x_{2}\right)= & : W\left(x_{1}, x_{2}\right) \\
W^{T}\left(x_{1}, x_{2}, x_{3}\right)= & : W\left(x_{1}, x_{2}, x_{3}\right) \\
W^{T}\left(x_{1}, x_{2}, x_{3}, x_{4}\right)= & : W\left(x_{1}, x_{2}, x_{3}, x_{4}\right)-W^{T}\left(x_{1}, x_{2}\right) W^{T}\left(x_{3}, x_{4}\right) \\
& -W^{T}\left(x_{1} ; x_{3}\right) W^{T}\left(x_{2} ; x_{4}\right)-W^{T}\left(x_{1} ; x_{4}\right) W^{T}\left(x_{2} ; x_{3}\right)
\end{aligned}
$$

usw.

Dabei haben die trunkierten Funktionen die Eigenschaften (W I) und (WIII) sowie den kleineren Träger:

mit

$$
\text { Träger } \widetilde{W}^{T}\left(k_{1}, \ldots, k_{n}\right) \leqq \bar{M}
$$

$\left(\mathrm{WII}^{\prime}\right) \quad \bar{M}=\left\{k: \sum_{j=1}^{r} k_{j} \in \frac{\circ}{V_{+}^{2 m}}\right.$ für $\left.r=1 ; \ldots ; n-1 ; \sum_{j=1}^{n} k_{j}=0\right\}$.

Schließlich haben die $\widetilde{W}^{T}\left(k_{1}, \ldots, k_{n}\right)$ nur noch eine einzige Vakuumsingularität, nämlich $\delta\left(\sum_{i=1}^{n} k_{i}\right)$ (Gesamtenergie-Impuls-Erhaltung), so daß die Auflösung von (5) nach $\widetilde{W}\left(k_{1}, \ldots, k_{n}\right)$ eine Entwicklung nach diesen Singularitäten darstellt. 
Während nun die Wightman-Funktionen zum Beispiel bei einer unendlichen raumartigen Separation der Argumente gegen eine Konstante gehen [13], verschwinden die trunkierten Vakuumerwartungswerte in diesem Fall stärker als jede Potenz [14], [15], [16].

Der nächste Schritt besteht darin, durch eine zu (5) analoge Entwicklung von den trunkierten Wightman-Funktionen den Beitrag der Massenschale zum Träger abzuspalten.

Infolge der Spektralzerlegung (1) und (2) bzw. der schwachen asymptotischen Konvergenz der aus den Haagschen quasilokalen Operatoren [11], [18], [19]

$$
\mathrm{B}\left[h_{n}\right]=\int d x_{1} d x_{2} \ldots d x_{n} h\left(x_{1}, \ldots, x_{n}\right) \mathrm{A}\left(x_{1}\right) \cdot \ldots \cdot \mathrm{A}\left(x_{n}\right)
$$

gebildeten $L S Z$-Operatoren [20] gelten die folgenden Aufspaltungen auf der Massenschale (Abschnitt IV):

$$
\begin{gathered}
\int d^{4} p g_{+}^{m}(p)\left\{\delta\left(p-\sum_{l=1}^{r} k_{l}\right) \widetilde{W}\left(k_{1}, \ldots, k_{n}\right)-\right. \\
\left.\widetilde{W}_{r+1}\left(k_{1}, \ldots, k_{r},-p\right)\left\langle\Phi_{p}^{m}\left|\tilde{\mathrm{A}}\left(k_{r+1}\right) \ldots \tilde{\mathrm{A}}\left(k_{n}\right)\right| 0\right\rangle\right\}=0 \\
\int d^{4} p g_{+}^{m}(p)\left\{\delta ( p - \sum _ { l = 1 } ^ { n } p _ { l } ) \delta ( p ^ { 2 } - m ^ { 2 } ) ( p ^ { 2 } - m ^ { 2 } ) \widetilde { W } \left(k_{1} ; \ldots k_{r}, \times\right.\right. \\
\left.\times p_{1}, \ldots p_{n}, q_{1} ; \ldots q\right)-\widetilde{W}\left(p_{1}, \ldots p_{n},-p\right) \times \\
\left.\quad \times\left[p^{2}-m^{2}\right] \widetilde{W}\left(k_{1}, \ldots k_{r}, p, q_{1}, \ldots q_{s}\right)\right\}=0 \\
\int d^{4} p g_{-}^{m}(p)\left\{\delta ( p - \sum _ { l = 1 } ^ { n } p _ { l } ) \delta ( p ^ { 2 } - m ^ { 2 } ) ( p ^ { 2 } - m ^ { 2 } ) \widetilde { W } \left(k_{1}, \ldots, k_{r}, \times\right.\right. \\
\left.\times p_{1}, \ldots p_{n}, q_{1}, \ldots q_{s}\right)-\widetilde{W}\left(-p, p_{1}, \ldots p_{n}\right) \times \\
\left.\times\left[p^{2}-m^{2}\right] \widetilde{W}\left(k_{1}, \ldots k_{r}, p, q_{1}, \ldots, q_{s}\right)\right\}=0 .
\end{gathered}
$$

Dabei ist $g_{ \pm}^{m}(p)$ eine Testfunktion, deren Träger in einer Umgebung der Massenschale im Vor- bzw. Rückwärtskegel konzentriert ist. Damit erwartet man eine Entwicklung der Art ${ }^{1}$

$$
\begin{aligned}
& W^{\mathrm{II}}\left(x_{1}, x_{2}, x_{3}, x_{4}\right)=W^{T}\left(x_{1}, x_{2}, x_{3}, x_{4}\right)- \\
& \quad \text { - Terme der Art }\left\{\int d z K\left(z \mid x_{i}\right) W^{T}\left(x_{1}, x_{2}, z\right) W^{T}\left(z, x_{3}, x_{3}\right)\right. \\
& \left.\quad+\text { Permutationen } \operatorname{der}\left(x_{j}, z\right)\right\}
\end{aligned}
$$

usw.

${ }^{1}$ Ich möchte Herrn Dr. H. J. Borchers für eine wertvolle Diskussion über die Art der zu erwartenden Entwicklung danken.

Commun. math. Phys., Vol. 2 
In diesen Gleichungen soll $K\left(z / x_{j}\right)$ ein geeigneter Integralkern sein. Die $W^{\mathrm{II}}\left(x_{1}, \ldots, x_{n}\right)$ sollen die Bedingungen (WI) und (WIII) erfüllen und die folgenden Trägereigenschaften im Impulsraum haben:

mit

$$
\text { Träger } \widetilde{W}^{\mathrm{II}}\left(k_{1}, \ldots, k_{n}\right) \leqq M^{\mathrm{II}}
$$

$\left(\mathrm{WII}^{\prime \prime}\right)$

$$
\begin{aligned}
M^{\mathrm{II}} & =\left\{k: k_{1} \in \frac{\circ}{V_{+}^{2 m}} ; \sum_{r=1}^{l} k_{r} \in \overline{V_{+}^{2 m}} \quad 2 \leqq l \leqq n-2 \times\right. \\
& \left.\times \sum_{r=1}^{n-1} k_{r} \in \frac{\circ}{V_{+}^{2 m}} ; \sum_{r=1}^{n} k_{r}=0\right\} .
\end{aligned}
$$

Durch Anwendung von Klein-Gordon-Operatoren $\prod_{l=1}^{n} K_{x_{l}}^{m}$ auf die $W^{\mathrm{II}}\left(x_{1}, \ldots, x_{n}\right)$ kann man dann sehr leicht Wightman-Funktionen erhalten, deren Träger im Impulsraum in allen Partialsummen $\sum_{r=1}^{l} k_{r}$ mit $1 \leqq l \leqq n-1 \mathrm{im}$ Vorkegel $V_{+}^{2 m}$ konzentriert ist. Vermutlich sind durch die Entwicklung (14) die Singularitäten der Wightman-Funktionen in irgendwelchen Partialsummen der Impulse auf der Massenschale auf solche in den einzelnen explizite auftretenden Impulsen $k_{i}$ zurückgeführt. Damit würden dann in den Funktionen $\prod_{l=1}^{n} K_{x_{l}}^{m} W_{\mathrm{II}}\left(x_{1}, \ldots, x_{n}\right)$ überhaupt keine Singularitäten auf der Massenschale mehr auftreten (Abschnitt III).

Über Entwicklungen der Art (8) können Modelle für lokale, lorentzinvariante Feldtheorien mit nichttrivialer S-Matrix konstruiert werden. Ein solches Modell erhält man, indem man von einem gewissen Index $N$ an alle $W^{\mathrm{II}}\left(x_{1}, \ldots, x_{n}\right)$ mit $n>N$ gleich Null setzt. Dann sind alle trunkierten Wightman-Funktionen "Produkte" von $W^{\text {II }}$-Funktionen mit einer Anzahl von Argumenten $n<N$. Die S-Matrix ist dann nichttrivial, falls die trunkierten Wightman-Funktionen noch Hauptwertsingularitäten auf der Massenschale enthalten. Leider ist die S-Matrix in diesen Modellen nicht unitär (Abschnitt IV).

In den folgenden Abschnitten wird eine solche Entwicklung für die Vierpunktfunktion hergeleitet.

$\mathrm{Zu}$ Gleichung (8) analoge Entwicklungen sind von SyManziK [22], [23] für die $r$-Funktionen und von ZimmermanN [24], [25] für die $\tau$-Funktionen hergeleitet worden. In Abschnitt II erweitern wir eine modifizierte Form der von Symanzik angegebenen Entwicklung auf die von SteinmanN [26], von Ruelle [27] sowie von Araki [8], [12] und BURGOYNE [29] eingeführten verallgemeinerten, retardierten Vierpunktfunktionen. 
In Abschnitt III benutzen wir das von denselben Autoren sowie Jost [25] angegebene Rekonstruktionsverfahren, um über die vierfachen Kommutatoren eine Entwicklung der Art (8) für die entsprechende Wightman-Funktion herzuleiten.

Die Abschnitte II und III sind formaler Natur, da die auftretenden retardierten Funktionen mathematisch nicht wohl definiert sind. Im Endergebnis (III.22) treten die retardierten Funktionen nicht mehr auf, sondern nur noch Matrixelemente von zweifachen retardierten Kommutatoren. Diese Matrixelemente werden im Anhang durch verallgemeinerte Jost-Lehmann-Dyson-Darstellungen präzise definiert.

Während in den Abschnitten II-III außer beim Beweis von Satz V nur die Voraussetzungen (AI)-(AIV) bzw. (WI)-(WIII) benutzt werden, wird in Abschnitt IV unter der weiteren Annahme der $L S Z$ Asymptotenbedingung die Aufspaltung (7) der Wightman-Funktionen auf der Massenschale hergeleitet und einige damit zusammenhängende Folgerungen über die Nichttrivialität der S-Matrix gezogen.

\section{Verallgemeinerte retardierte Funktionen}

Die $r$-Funktionen [20] sind die Vakuumerwartungswerte der $n$-fachen retardierten Operatorprodukte (über die Mehrdeutigkeiten bei der Definition dieser Produkte [32] werden in Abschnitt III noch einige kurze Bemerkungen gemacht):

$$
\begin{gathered}
R\left(x / x_{1}, \ldots, x_{n}\right)=:(-i)^{n} \sum_{\text {Perm. }\{1, \ldots, n\}} \theta\left(x-x_{1}\right) \theta\left(x_{1}-x_{2}\right) \ldots \theta\left(x_{n-1}-x_{n}\right) \times \\
\left.\left.\quad \times\left[\ldots\left[\mathrm{A}(x) ; \mathrm{A}\left(x_{1}\right)\right] ; \mathrm{A}\left(x_{2}\right)\right] ; \ldots\right] ; \mathrm{A}\left(x_{n}\right)\right] \\
\quad r\left(x / x_{1}, \ldots, x_{n}\right)=\left\langle 0\left|R\left(x / x_{1}, \ldots, x_{n}\right)\right| 0\right\rangle .
\end{gathered}
$$

Die $r$-Funktionen sind symmetrisch in den Argumenten $x_{1} ; \ldots ; x_{n}$ und invariant unter der Poincaré-Gruppe $i L g_{+}^{\top}$.

$$
r\left(\Lambda x+a / \ldots, \Lambda x_{i}+a, \ldots\right)=r\left(x / \ldots, x_{i}, \ldots\right)(\Lambda ; a) \in i L g_{+}^{\nearrow} .
$$

Außerdem gilt die Trägerbedingung:

$$
r\left(x / x_{1}, \ldots, x_{n}\right)=0 \text { falls }\left(x-x_{j}\right) \notin \overline{V_{+}^{0}} \quad \text { für ein } x_{j} .
$$

Schließlich gelten für die $R$-Produkte (1) die Operatoridentitäten: [34], [35].

$$
\begin{aligned}
& R\left(x / y, z_{1}, \ldots, z_{n}\right) \\
&=-i \theta(x-y) \sum_{\text {Combin. }}\left[R\left(x / z_{1}, \ldots, z_{k}\right) ; R\left(y / z_{k+1}, \ldots, z_{n}\right)\right] \\
& R\left(x / y, z_{1}, \ldots, z_{n}\right)-R\left(y / x, z_{1}, \ldots, z_{n}\right) \\
&=-i \sum_{\text {Combin. }}\left[R\left(x / z_{1}, \ldots z_{k}\right) ; R\left(y / z_{k_{1}+1}, \ldots, z_{n}\right)\right]
\end{aligned}
$$


Mit den Sätzen von Schwartz [6], von Hall und Wightman [7], von Tomozawa [10] und dem verallgemeinerten Edge-of-the-WedgeTheorem von EPstein [8], [35] ergeben sich aus den Eigenschaften (9)-(14) Regularitätseigenschaften für die Fourier-Laplace-Transformierten, die definiert sind durch:

$$
\left\{\begin{array}{c}
\tilde{r}\left(p / p_{1}, \ldots, p_{n}\right)=: \frac{1}{(2 \pi)^{5 / 2(n+1)}} \int d^{4} x \prod_{j=1}^{n} d^{4} x_{j} e^{i p x+i \sum_{j=1}^{n} p_{j} x_{j}} \times \\
\times r\left(x / x_{1}, \ldots, x_{n}\right) \\
=\frac{1}{(2 \pi)^{5 / 2(n+1)-4}} \delta\left(p+\sum_{j=1}^{n} p_{j}\right) \int \prod_{j=1}^{n} d \xi_{j} e^{-i \sum_{j=1}^{n} \xi_{j} p_{j}} r^{\prime}\left(\xi_{1}, \ldots, \xi_{n}\right) \\
\tilde{r}\left(p / p_{1}, \ldots, p_{n}\right)=\delta\left(p+\sum_{j=1}^{n} p_{j}\right) \tilde{r}^{\prime}\left(p / p_{1}, \ldots, p_{n}\right) \\
\xi_{j}=x-x_{j} ; \quad p=k+i q \quad k ; q \text { reell } .
\end{array}\right\}
$$

Diese bekannten Eigenschaften sollen für die Drei- und Vierpunktfunktion in den beiden folgenden Sätzen zusammengefaßt werden: Dafür ist es zweckmäßig, einige neue Beziehungen und Größen einzuführen:

$$
\begin{aligned}
r_{j}(x)_{3} & \equiv r_{j}\left(x_{1}, x_{2}, x_{3}\right) \equiv r\left(x_{j} / x_{m}, x_{n}\right) \\
r_{j}(x) & \equiv r_{j}\left(x_{1}, x_{2}, x_{3}, x_{4}\right) \equiv r\left(x_{j} / x_{m}, x_{n}, x_{s}\right) .
\end{aligned}
$$

Im folgenden bedeuten $(j, m, n)$ oder $(j, m, n, s)$ immer eine Permutation von $(1,2,3)$ bzw. $(1,2,3,4)$. Neben den retardierten Funktionen führen wir die advancierten Funktionen

$$
a_{j}(x)=r_{j}(-x)
$$

und im Falle der Vierpunktfunktionen die folgenden gemischten oder verallgemeinerten $r$-Funktionen [26]-[29] ein:

mit:

$$
\begin{aligned}
& r_{j k}(x)=: r_{j}(x)+i S_{j k}(x) \\
& a_{j k}(x)=: a_{j}(x)+i S_{j k}(-x)
\end{aligned}
$$

$$
S_{j k}(x)=:\left\langle 0\left|\left[R\left(x_{j} \mid x_{m}, x_{n}\right) ; A\left(x_{k}\right)\right]\right| 0\right\rangle .
$$

Die gemischten Funktionen $r_{j k}(x) ; a_{j k}(x)$ sind ebenfalls Lorentzinvariant, symmetrisch in $x_{m}$ und $x_{n}$, und sie haben die Trägereigenschaften:

$$
\begin{array}{rlll}
r_{j k}(x)=0 \quad \text { für } \quad & \left(x_{j}-x_{m}\right) \notin \overline{V_{ \pm}^{0}} ; \quad \text { oder } \quad\left(x_{j}-x_{n}\right) \notin \overline{V_{ \pm}^{0}} ; \\
& \text { oder }\left\{\left(x_{k}-x_{m}\right) \notin \overline{V_{ \pm}^{0}} \quad \text { und } \quad\left(x_{k}-x_{n}\right) \notin \overline{V_{ \pm}^{0}}\right\} .
\end{array}
$$

Schließlich definieren wir die 8- bzw. 12-dimensionalen, offenen, reellen 
Über die Einteilchensingularitäten in Relativistischen Quantenfeldtheorien 183

Kegel im Raum des Imaginärteils von $p$ durch:

$$
\begin{gathered}
\mathscr{C}_{i}^{3} \pm=:\left\{q=\left(q_{1} ; q_{2} ; q_{3}\right): \sum_{r=1}^{3} q_{r}=0 ; q_{j} \in V_{ \pm}^{0} ; q_{s} \in V_{ \pm}^{0}\right\} \\
\mathscr{C}_{i}^{ \pm}=:\left\{q=\left(q_{1} ; q_{2} ; q_{3} ; q_{4}\right): \sum_{r=1}^{4} q_{r}=0 ; q_{m} \in V_{ \pm}^{0} ; q_{n} \in V_{ \pm}^{0} ; q_{j} \in V_{ \pm}^{0}\right\} \\
\mathscr{C}_{i j}^{ \pm}=:\left\{q=\left(q_{1} ; q_{2} ; q_{3} ; q_{4}\right): \sum_{r=1}^{4} q_{r}=0 ; q_{j} \in V_{ \pm}^{0} ;\left(q_{j}+q_{n}\right) \in V_{ \pm}^{0} ; \times\right. \\
\left.\times\left(q_{j}+q_{m}\right) \in V_{ \pm}^{0}\right\}
\end{gathered}
$$

sowie die reellen Punktmengen $E_{j}^{3}(a), E_{j}(a)$ und $E_{i j}(a)$ durch:

$$
\begin{gathered}
E_{j}^{3}\left(a^{2}\right)=\left\{k=\left(k_{1} ; k_{2} ; k_{3}\right): \sum_{r=1}^{3} k_{r}=0 ; k_{j}^{2}>a^{2}\right\} \\
E_{j}\left(a^{2}\right)=\left\{k=\left(k_{1} ; k_{2} ; k_{3} ; k_{4}\right): \sum_{r=1}^{4} k_{r}=0 ; k_{j}^{2}>a^{2}\right\} \\
E_{i j}\left(a^{2}\right)=\left\{k=\left(k_{1} ; k_{2} ; k_{3} ; k_{4}\right): \sum_{r=1}^{4} k_{r}=0 ;\left(k_{i}+k_{j}\right)^{2}>a^{2}\right\} .
\end{gathered}
$$

Die Kegel $\mathscr{C}_{\alpha}^{\beta}$ sind gerade so definiert, daß gilt:

1. $\mathscr{C}_{i}^{3+}$ und $\mathscr{C}_{j}^{3-}$ unterscheiden sich nur im Vorzeichen von $q_{s}^{0}$.

2. $\mathscr{C}_{i}^{ \pm}$und $\mathscr{C}_{i j}^{ \pm}$unterscheiden sich nur im Vorzeichen von $q_{j}^{0}$.

3. $\mathscr{C}_{i j}^{+}$und $\mathscr{C}_{m n}^{-}$unterscheiden sich nur im Vorzeichen von $q_{j}^{0}+q_{n}^{0}$.

Weiterhin bezeichne $L_{+}(c)$ die homogene, komplexe Lorentzgruppe mit der Determinante $+1, \operatorname{conv}\{M\}$ die konvexe Hülle der Menge $M$ und C $M$ die Komplementärmenge von $M$.

Mit diesen Definitionen gilt dann [10], [12], [26]-[30]:

«) Dreipunktfunktion:

Satz I. Die sechs Dreipunktfunktionen $\tilde{r}_{j}^{\prime}(k)$ und $\tilde{a}_{j}^{\prime}(k)$ sind Grenzwerte einer eindeutigen, analytischen Funktion $g(p)$ der Komplexen Veränderlichen $p=k+i q\left[k=\left(k_{1}, k_{2}, k_{3}\right) ; q=\left(q_{1}, q_{2}, q_{3}\right)\right.$ reell $]$ :

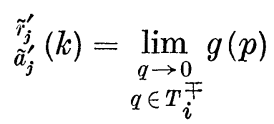

$g(p)$ ist eindeutig und analytisch in der einfach zusammenhängenden Punktmenge $\mathrm{N}_{3}$, bestehend aus der Vereinigung aller möglichen erweiterten Röhren

$$
\mathfrak{R}_{j}^{ \pm}=\bigcup_{\Lambda} \Lambda T_{j}^{\ddagger} ; \quad \Lambda \in L_{+}(c)(j=1,2,3)
$$

mit

$$
T_{j}^{ \pm}=\left\{p: \sum_{r=1}^{3} p_{r}=0 ; q \in \mathscr{C}_{j}^{3 \pm}\right\} .
$$


$g(p)$ hat analytische Fortsetzungen in die Punktmengen

$$
\Sigma_{i j}^{3}\left(m^{2}\right)=:\left\{p: \sum_{r=1}^{3} p_{r}=0 ; p \in \text { conv. }\left\{T_{i}^{+} \cup T_{i}^{-}\right\} \cap C E_{s}\left(m^{2}\right)\right\}
$$

und in die reellen Punkte

Außerdem gilt:

$$
B_{i j}\left(m^{2}\right)=:\left\{p: \sum_{r=1}^{3} p_{r}=0 ; q=0 ; k_{i}^{2}<m^{2} ; k_{j}^{2}<m^{2}\right\} .
$$

$$
\begin{gathered}
\tilde{\pi}_{\tilde{a}_{j}^{\prime}}^{\prime}(p) \equiv g(p) \quad \text { für } \quad p \in T_{j}^{\mp} \\
g(p)=g(\Lambda p) \quad \text { für } \quad p \in T_{j}^{ \pm} \text {und } \Lambda \in L_{+}(c)
\end{gathered}
$$

und

$$
e^{-B t} \mid g(k+i(Q+t q) \mid<\text { Polynom }\{k\}
$$

für jeden Strahl $Q+t q(t>0)$, der ganz in einem der $T_{j}^{ \pm}$verläuft und für $B>0$; sowie

$$
\tilde{r}_{j}^{\prime}(k)-\tilde{a}_{i}^{\prime}(k)=0 \quad \text { für } \quad k_{s}^{2}<m^{2} .
$$

Aus diesem Satz ergibt sich mit Hilfe des verallgemeinerten Edgeof-the-Wedge-Theorems von Epstein [8], [36] die für alle weiteren Überlegungen wichtige Folgerung:

Folgerung $\mathbf{F I}_{1}$ : Die drei Funktionen

$$
g_{s}(p)=:\left(p_{s}^{2}-m^{2}\right) g(p) \quad s=1,2,3
$$

sind in den größeren Bereichen

regulär, und es gilt:

$$
N_{3}^{s}=N_{3} \cup \Sigma_{i j}^{3}\left(4 m^{2}\right)
$$

$$
\left(k_{s}^{2}-m^{2}\right)\left[\tilde{r}_{j}^{\prime}(k)-\tilde{a}_{i}^{\prime}(k)\right]=0 \quad \text { für } \quad k_{s}^{2}<4 m^{2} .
$$

Beweis: Es genügt die Gleichung (32) zu beweisen. Denn die Aussage über den Regularitätsbereich folgt daraus mit Satz I und dem verallgemeinerten Edge-of-the-Wedge-Theorem, da sich die beiden Röhren $T_{j}^{-}$und $T_{i}^{+}$nur durch das Vorzeichen von $q_{s}^{0}$ unterscheiden [28].

Aus den Definitionsgleichungen (9), (10) und (16) folgt:

$$
\begin{aligned}
& \tilde{r}_{j}(k)-\tilde{a}_{i}(k)=\frac{1}{(2 \pi)^{5}} \int d^{4} x e^{i k x} \theta\left(x_{j}-x_{i}\right) \times \\
& \times\left\{\left\langle 0\left|\left[A\left(x_{j}\right) ; \mathrm{A}\left(x_{i}\right)\right] \mathrm{T}\left(x_{s}\right) \mathrm{A}(0)\right| 0\right\rangle-\left\langle 0\left|\mathrm{~A}(0) \mathrm{T}\left(-x_{s}\right)\left[\mathrm{A}\left(x_{j}\right) ; \mathrm{A}\left(x_{i}\right)\right]\right| 0\right\rangle .\right.
\end{aligned}
$$

Einsetzen der Spektralzerlegung (5); (6) für den Translationsoperator und Integration über $x_{s}$ ergibt mit $\langle 0|\mathrm{~A}(0)| 0\rangle=0$ sofort die Behauptung.

$\beta)$ Vierpunktfunktion:

Satz II. Die zweiunddreißig Vierpunktfunktionen

$$
\tilde{r}_{j}^{\prime}(k) ; \tilde{a}_{j}^{\prime}(k) ; \tilde{r}_{i j}^{\prime}(k) \text { und } a_{i j}^{\prime}(k)
$$


Über die Einteilchensingularitäten in Relativistischen Quantenfeldtheorien 185 sind Grenzwerte einer regulären Funktion $G(p)$ der komplexen Veränderlichen

$$
\begin{gathered}
p=k+i q\left[k=\left(k_{1} ; k_{2} ; k_{3} ; k_{4}\right), q=\left(q_{1} ; q_{2} ; q_{3} ; q_{4}\right) \text { reell } ; \sum_{r=1}^{4} p_{r}=0\right] \\
\tilde{r}_{i}^{\prime}(k)=\lim _{\substack{q \rightarrow 0 \\
q \in T_{i}^{\mp}}} G(k+i q) \quad\{8\} \\
\tilde{a}_{i}^{\prime}(k)=\lim _{\substack{q \rightarrow 0 \\
q \in T_{i j}^{\mp}}}^{\mp} G(k+i q) \quad\{24\}
\end{gathered}
$$

$G(p)$ ist eindeutig und regulär in der einfach zusammenhängenden Punktmenge $\mathrm{N}_{4}$, bestehend aus der Vereinigung aller möglichen erweiterten Röhren $\mathfrak{R}_{i}^{ \pm} ; \mathfrak{R}_{i j}^{ \pm}$mit

$$
\left\{\begin{array}{l}
\mathfrak{R}_{i}^{ \pm}=\bigcup_{\Lambda} \Lambda T_{i}^{ \pm} ; \mathfrak{R}_{i j}^{ \pm}=\bigcup_{\Lambda} \Lambda T_{i j}^{ \pm} ; \Lambda \in L_{+}(c) \\
T_{i}^{ \pm}=\left\{p: \sum_{r=1}^{4} p_{r}=0 ; q \in \mathscr{C}_{i}^{ \pm}\right\} \\
T_{i j}^{ \pm}=\left\{p: \sum_{r=1}^{4} p_{r}=0 ; q \in \mathscr{C}_{i j}^{ \pm}\right\}
\end{array}\right\}
$$

$G(p)$ hat analytische Fortsetzungen in die Punktmengen

$$
\left\{\begin{array}{l}
\Sigma_{i j}^{+}\left(m^{2}\right)=\left\{p: p \in \operatorname{conv}\left\{T_{i}^{ \pm} \cup T_{i j}^{ \pm}\right\} \cap C E_{j}\left(m^{2}\right)\right\} \\
\sum_{i j}^{m n}\left(m^{2}\right)=\left\{p: p \in \operatorname{conv} .\left\{T_{i j}^{-} \cup T_{m n}^{+}\right\} \cap C E_{j n}\left(m^{2}\right)\right\}
\end{array}\right\}
$$

und in die reellen Punkte

$$
\begin{aligned}
B\left(m^{2} ; m^{2}\right)=\left\{p: \sum_{r=1}^{4} p_{r}\right. & =0 ; q=0 ; k_{n}^{2}<m^{2} ;\left(k_{i}+k_{j}\right)^{2}<m^{2} ; 1 \leqq \\
& \leqq n ; i ; j \leqq 4\} .
\end{aligned}
$$

Außerdem gilt:

$$
\begin{aligned}
& \left\{\begin{array}{l}
\tilde{r}_{i}^{\prime}(p) \equiv G(p) \text { für } p \in T_{i}^{\mp} ; \tilde{a}_{i j}^{\prime}(p) \equiv G(p) \text { für } \quad p \in T_{i j}^{\mp} \\
G(p)=G(\Lambda p) \quad \text { für } \quad p \in T_{i}^{ \pm} ; T_{i j}^{ \pm} \quad \text { und } \quad \Lambda \in L_{+}(c)
\end{array}\right\} \\
& l^{-B t}|G(k+i(Q+t q))|<\text { Polynom }\{k\}
\end{aligned}
$$

für $B>0$ und jeden Strahl $Q+t q(t \geqq 0)$, der ganz in einer der Röhren $T_{i}^{ \pm}$oder $T_{i j}^{ \pm}$verläuft.

Schließlich gelten die Identitäten:

$$
\begin{gathered}
\tilde{r}_{i}^{\prime}(k)-\tilde{r}_{i j}^{\prime}(k)=0 \quad \text { für } \quad k_{j}^{2}<m^{2} \\
\tilde{a}_{i j}^{\prime}(k)=0 \quad \text { für } \quad\left(k_{j}+k_{n}\right)^{2}<m^{2} \\
\tilde{r}_{i j}^{\prime}(k)-\tilde{a}_{m n}^{\prime}(k)=0 . \\
\tilde{r}_{i j}^{\prime}(k)+\tilde{r}_{j i}^{\prime}(k)-\tilde{a}_{m n}^{\prime}(k)-\tilde{a}_{n m}^{\prime}(k)=0 .
\end{gathered}
$$


Eine zu $F I_{1}$ analoge Folgerung lautet für den Fall der Vierpunktfunktion:

Folgerung FII $_{1}$ : Die sieben Funktionen

$$
\left\{\begin{aligned}
G_{j}(p) & =:\left(p_{j}^{2}-m^{2}\right) G(p) \\
G_{j n}(p) & =:\left[\left(p_{j}+p_{n}\right)^{2}-m^{2}\right] G(p)
\end{aligned}\right\}
$$

sind in den größeren Bereichen

regulär, und es gilt:

$$
\left.\begin{array}{l}
N_{4}^{i \pm}=N_{4} \cup \Sigma_{i j}^{ \pm}\left(4 m^{2}\right) \quad b z w . \\
N_{4}^{j n}=N_{4} \cup \Sigma_{i j}^{m n}\left(4 m^{2}\right)
\end{array}\right\}
$$

$$
\begin{aligned}
& \left(k_{j}^{2}-m^{2}\right)\left[\begin{array}{l}
\tilde{r}_{i}^{\prime} \\
\tilde{a}_{i}^{\prime}
\end{array}(k)-\tilde{r}_{i j}^{\prime}(k)\right]=0 \quad \text { für } \quad k_{j}^{2}<4 m^{2} \\
& {\left[\left(k_{j}-k_{n}\right)^{2}-m^{2}\right]\left[\tilde{r}_{i j}^{\prime}(k)-a_{m n}^{\prime}(k)\right]=0 \text { für }\left(k_{j}+k_{n}\right)^{2}<4 m^{2} \text {. }}
\end{aligned}
$$

Der Beweis dieser Folgerung ergibt sich wieder mit Hilfe des Edgeof-the-Wedge-Theorems aus Satz II und den letzten beiden Gleichungen, die mittels der Operatoridentität (14), den Steinmann-Relationen (41) und der Spektralzerlegung des Translationsoperators hergeleitet werden können (siehe Beweis zu Satz III.) Da dieser Beweis etwas umständlich ist, und die Folgerung $\mathrm{FII}_{1}$ nicht mehr benutzt werden wird (sie würde erst bei der Fünfpunktfunktion eine Rolle spielen), soll er hier übergangen werden.

Wir definieren nun 32 neue Funktionen durch:

$$
\begin{aligned}
& { }_{a_{j}}^{r_{j}}(x)^{\mathrm{I}}=:{ }_{a_{j}}^{r_{j}}(x)+\int d^{4} z\left\{\begin{array}{c}
r \\
a
\end{array}\left(x_{j} / x_{s}, z\right) K_{z}^{m} \underset{a}{r}\left(z / x_{n}, x_{i}\right)+\right. \\
& \left.+{ }_{a}^{r}\left(x_{j} \mid x_{n}, z\right) K_{z}^{m} \underset{a}{r}\left(z / x_{s}, x_{i}\right)+{ }_{a}^{r}\left(x_{j} \mid x_{i}, z\right) K_{z}^{m} \underset{a}{r}\left(z / x_{n}, x_{s}\right)\right\} \\
& { }_{a_{j i}}^{r_{j i}}(x)^{\mathrm{I}}=:{ }_{a_{j i}}^{r_{j i}}(x)+\int d^{4} z\left\{\begin{array}{l}
a \\
r
\end{array}\left(z / x_{i}, x_{j}\right) K_{z}^{m}{ }_{n}^{r}\left(z / x_{s}, x_{n}\right)+\right. \\
& \left.+{ }_{a}^{r}\left(x_{j} \mid x_{s}, z\right) K_{z}^{m a}\left(x_{n} \mid x_{i}, z\right)+{ }_{a}^{r}\left(x_{j} \mid x_{n}, z\right) K_{z}^{m a}\left(x_{s} \mid x_{i}, z\right)\right\} .
\end{aligned}
$$

Dabei ist $K_{z}^{m}$ der Klein-Gordon-Operator zur Masse $m$. Die Funktionen $r_{j}(x)^{\mathrm{I}}, a_{j}(x)^{\mathrm{I}}$ in Gl. (46) unterscheiden sich von den entsprechenden Funktionen von Symanzik [22], [23] dadurch, daß hier als Integralkern die Reziproke $K_{z}^{m}$ der freien retardierten (advancierten) Zweipunktfunktion auftritt, während bei SymanziK die Reziproke der vollen Zweipunktfunktion abgespalten wird (s. Diskussion am Ende von Abschnitt III).

Die neuen Funktionen $r(x)^{\mathrm{I}}, a(x)^{\mathrm{I}}$ haben dieselben Invarianz- und Trägereigenschaften Gl. (12) bzw. (20) wie die ursprünglichen Funktionen. Daraus folgt aber, daß ihre Fourier-Transformierten Randwərte einer in $N_{4}$ eindeutigen und regulären Funktion sind, und dort die Beschränktheitsbedingungen (38) erfüllt sind. Wir werden nun mit dem Beweis des folgenden Satzes diesen Regularitätsbereich vergrößern und drei zu Gl. (39)-(41) analoge Identitäten herleiten. 
Über die Einteilchensingularitäten in Relativistischen Quantenfeldtheorien 187

Satz III. Die zweiunddreißig Funktionen $\tilde{r}_{j}^{\prime}(k)^{\mathrm{I}}, \tilde{a}_{j}^{\prime}(k)^{\mathrm{I}}, \tilde{r}_{i j}^{\prime}(k)^{\mathrm{I}}$ und $\tilde{a}_{i j}^{\prime}(k)^{\mathrm{I}}$ sind Grenzwerte einer regulären Funktion $G(p)^{\mathrm{I}}$ der komplexen Veränderlichen $p=k+i q, k ; q$ reell:

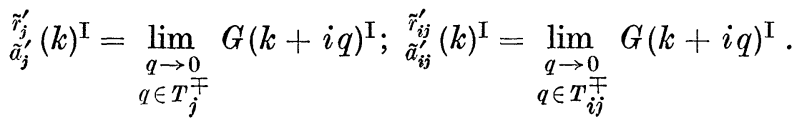

$G(p)^{\mathrm{I}}$ ist eindeutig und regulär in der einfach zusammenhängenden Punktmenge $\mathrm{N}_{4}$, bestehend aus der Vereinigung aller möglichen erweiterten Röhren $\mathfrak{R}_{i}^{ \pm} ; \mathfrak{R}_{i j}^{ \pm}$, und hat analytische Fortsetzungen in die Punktmengen

$$
\begin{gathered}
\sum_{i j}^{+}\left(m^{2}\right)=\left\{p: p \in \mathrm{conv} \cdot\left\{T_{i}^{ \pm} \cup T_{i j}^{ \pm}\right\} \cap C E_{j}\left(m^{2}\right)\right\} \\
\sum_{i j}^{m n}\left(4 m^{2}\right)=\left\{p: p \in \mathrm{conv} \cdot\left\{T_{i j}^{-} \cup T_{m n}^{+}\right\} \cap C E_{j n}\left(4 m^{2}\right)\right\}
\end{gathered}
$$

sowie in die reellen Punkte

$$
\left.\begin{array}{r}
B\left(m^{2} ; 4 m^{2}\right)=\left\{p: \sum_{r=1}^{4} p_{r}=0 ; q=0 ; k_{n}^{2}<m^{2} ;\left(k_{n}+k_{j}\right)^{2}<4 m^{2} ;\right. \\
1 \leqq n ; j \leqq 4
\end{array}\right\}
$$

Außerdem gelten die Relationen (37) und (38) für $r(p)^{\mathrm{I}}$ und $G(p)^{\mathrm{I}}$ sowie die Identitäten:

$$
\begin{gathered}
\tilde{r}_{i}^{\prime}(k)^{\mathrm{I}}-\tilde{r}_{i j}^{\prime}(k)^{\mathrm{I}}=0 \quad \text { für } \quad k_{j}^{2}<m^{2} \\
\tilde{a}_{i j}^{\prime}(k) \quad \text { für } \quad\left(k_{j}+k_{n}\right)^{2}<4 m^{2} \\
\tilde{r}_{i j}^{\prime}(k)^{\mathrm{I}}-\tilde{a}_{m n}^{\prime}(k)^{\mathrm{I}}=0 \quad{ }^{\mathrm{I}}=0 . \\
\tilde{r}_{i j}^{\prime}(k)^{\mathrm{I}}+\tilde{r}_{j i}^{\prime}(k)^{\mathrm{I}}-\tilde{a}_{m n}^{\prime}(k)^{\mathrm{I}}-\tilde{a}_{n m}^{\prime}(k)^{\mathrm{I}}=0 .
\end{gathered}
$$

Beweis: Wie schon erwähnt wurde, ergeben sich die Regularität in $N_{4}$ sowie die Relationen (37) und (38) in bekannter Weise [10]; [12]; [26]-[30] aus den Invarianz- und Trägereigenschaften im Ortsraum.

Für $k_{j}^{2}<m^{2}$ folgt durch Fouriertransformation aus den Gleichungen (46) und (47) mit (39):

$$
\begin{aligned}
& \begin{array}{l}
\tilde{r}_{i}^{\prime} \\
\tilde{a}_{i}^{\prime}
\end{array}(k)^{\mathrm{I}}-\tilde{r}_{i j}^{\prime}(k)^{\mathrm{I}}=2 \pi\left\{\begin{array}{l}
\tilde{r}_{i j}^{\prime} \\
\tilde{a}^{\prime}
\end{array}\left(k_{i} / k_{m}, k_{n}+k_{j}\right) \times\right. \\
& \times\left(\left(k_{n}+k_{j}\right)^{2}-m^{2}\right)\left[\frac{\tilde{r}^{\prime}}{\tilde{a}^{\prime}}\left(-k_{n}-k_{j} \mid k_{n}, k_{j}\right)-\underset{\tilde{r}^{\prime}}{\tilde{a}^{\prime}}\left(k_{n} / k_{j}-k_{n}-k_{j}\right)\right]+ \\
& +\tilde{a}^{\prime}\left(k_{i} / k_{n}, k_{m}+k_{j}\right)\left(\left(k_{m}+k_{j}\right)^{2}-m^{2}\right) \times \\
& \times\left[\begin{array}{l}
\tilde{r}^{\prime} \\
\tilde{a}^{\prime}
\end{array}\left(-k_{m}-k_{j} / k_{m}, k_{j}\right)-\tilde{r}_{\tilde{r}^{\prime}}^{\tilde{a}^{\prime}}\left(k_{m} / k_{j},-k_{m}-k_{j}\right)\right]+
\end{aligned}
$$

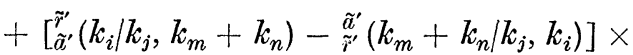

$$
\begin{aligned}
& \left.\times\left(\left(k_{m}+k_{n}\right)^{2}-m^{2}\right) \stackrel{\tilde{r}^{\prime}}{\tilde{a}^{\prime}}\left(-k_{m}-k_{n} / k_{m}, k_{n}\right)\right\} \text {. }
\end{aligned}
$$

Daraus ergibt sich sofort mit Satz I Gl. (29) die Gleichung (50), da für $k_{j}^{2}<m^{2}$ die eckigen Klammern verschwinden. 
Es sei nun $Z_{i j}\left(\varepsilon / m^{2}\right)$ eine komplexe Umgebung des Abschnittes der reellen Achse mit $k_{j}^{2}<m^{2}$ :

$$
Z_{i j}\left(\varepsilon / m^{2}\right)=\left\{p: \sum_{r=1}^{4} p_{r}=0 ;|q|<\varepsilon ; k_{j}^{2}<m^{2}\right\} .
$$

Dann ist $\underset{\tilde{a}_{i}^{\prime}}{\tilde{r}_{i}^{\prime}}(p)^{\mathrm{I}}$ regulär für $p \in \mathfrak{D}_{i}^{\mp}$

$$
\mathfrak{D}_{i}^{\mp}=: Z_{i j}\left(\varepsilon / m^{2}\right) \cap T_{i}^{\mp}
$$

und $\tilde{a}_{i j}^{\prime}(p)^{\mathrm{I}}$ regulär für $p \in \mathfrak{S}_{i j}^{\mp}$

$$
\mathfrak{S}_{i j}^{\mp}=: Z_{i j}\left(\varepsilon / m^{2}\right) \cap T_{i j}^{\mp} .
$$

Da sich $T_{i}$ und $T_{i j}$ nur im Vorzeichen von $q_{j}^{0}$ unterscheiden, so folgt mit den Gleichungen (50); (55) und (56) aus dem verallgemeinerten Edge-ofthe Wedge-Theorem [8]; [36], daß

$$
\tilde{r}_{i}^{\prime}(p)^{\mathrm{I}}\left[\tilde{a}_{i}^{\prime}(p)^{\mathrm{I}}\right] \text { und } \tilde{r}_{i j}^{\prime}(p)^{\mathrm{I}}\left[\tilde{a}_{i j}^{\prime}(p)^{\mathrm{I}}\right]
$$

eine gemeinsame analytische Fortsetzung in den Durchschnitt $B_{i j}^{+}$von $Z_{i j}\left(\varepsilon / m^{2}\right)$ und der abgeschlossenen konvexen Hülle von $T_{i j}^{-} \cup T_{i}^{-}$bzw. $T_{i j}^{+} \cup T_{i}^{+}$haben:

$$
B_{i j}^{ \pm}=Z_{i j}\left(\varepsilon / m^{2}\right) \cap \overline{\operatorname{conv}\left\{T_{i j}^{+} \cup T_{i}^{ \pm}\right\}} .
$$

Die Holomorphie-Envelope von $T_{i j}^{ \pm} \cup T_{i}^{ \pm} \cup B_{i j}^{ \pm}$ist nach einem Satz von Bros; Erstein und Glaser [28] gerade die Punktmenge $\Sigma_{i j}^{ \pm}\left(m^{2}\right)$.

Die Steinmann-Relation (52) folgt unmittelbar durch Einsetzen von (46) und (47) in die entsprechende Relation (41) aus Satz II, da sich die auftretenden Terme aus Produkten von Dreipunktfunktionen bei Berücksichtigung der Trägerbedingung $\sum_{r=1}^{4} k_{r}=0$ gegenseitig kompensieren.

Als nächstes soll die Gleichung (51) bewiesen werden. Infolge der Steinmann-Relation (52) genügt es, den Beweis für die Größe

$$
A(k)=:\left[\tilde{r}_{i j}(k)^{\mathrm{I}}-\tilde{a}_{m n}(k)^{\mathrm{I}}\right]-\left[\tilde{r}_{j i}(k)^{\mathrm{I}}-\tilde{a}_{n m}(k)^{\mathrm{I}}\right]
$$

zu führen.

Aus den Gleichungen (46) und (47) folgt für $A(k)$ :

$$
\begin{aligned}
A(k) & =I(k)-4 \pi \delta\left(\sum_{r=1}^{4} k_{r}\right) \times \\
& \times\left\{\tilde{r}^{\prime}\left(k_{i} / k_{m}, k_{j}+k_{n}\right)\left[\left(k_{j}+k_{n}\right)^{2}-m^{2}\right] \tilde{a}^{\prime}\left(k_{n} / k_{j},-k_{n}-k_{j}\right)\right. \\
& \left.-\tilde{r}^{\prime}\left(k_{j} / k_{n}, k_{\imath}+k_{m}\right)\left[\left(k_{i}+k_{m}\right)^{2}-m^{2}\right] \tilde{a}^{\prime}\left(k_{m} / k_{i},-k_{m}-k_{i}\right)\right\},
\end{aligned}
$$

wobei $I(k)$ definiert ist durch:

$$
I(k)=:\left[\tilde{r}_{i j}(k)-\tilde{a}_{m n}(k)\right]-\left[\tilde{r}_{j i}(k)-\tilde{a}_{n m}(k)\right] .
$$


Berücksichtigen wir die Definitionsgleichungen (17)-(19) für $\tilde{r}_{i j} \mathrm{bzw}$. $\tilde{a}_{m n}$, so folgt mit der Operatorenidentität (14) für $I(k)$ :

$$
\begin{aligned}
I(k) & =-\frac{i}{(2 \pi)^{10}} \int d^{4} x e^{i k x}\left\{\left\langle 0\left|\left[R\left(x_{i} \mid x_{m}\right) ; R\left(x_{j} \mid x_{n}\right)\right]\right| 0\right\rangle-\right. \\
& \left.-\left\langle 0\left|\left[R\left(-x_{m} \mid-x_{i}\right) ; R\left(-x_{n} \mid-x_{j}\right)\right]\right| 0\right\rangle\right\}- \\
& -\frac{i}{(2 \pi)^{10}} \int d^{4} x e^{i k x}\left\{\left\langle 0\left|\left[R\left(x_{i} \mid x_{n}\right) ; R\left(x_{j} \mid x_{m}\right)\right]\right| 0\right\rangle+\right. \\
& \left.+\left\langle 0\left|\left[R\left(-x_{n} \mid-x_{i}\right) ; R\left(-x_{m} \mid-x_{j}\right)\right]\right| 0\right\rangle\right\} .
\end{aligned}
$$

Infolge der Jakobi-Identität

$\left[\left[\left[\mathrm{A}\left(x_{i}\right) ; \mathrm{A}\left(x_{j}\right)\right] ; \mathrm{A}\left(x_{s}\right)\right] ; \mathrm{A}\left(x_{r}\right)\right]-\left[\left[\left[\mathrm{A}\left(x_{i}\right) ; \mathrm{A}\left(x_{j}\right)\right] ; \mathrm{A}\left(x_{s}\right)\right] ; \mathrm{A}\left(x_{r}\right)\right]$

und der $P C T$-Symmetrie:

$$
=\left[\left[\mathrm{A}\left(x_{i}\right) ; \mathrm{A}\left(x_{j}\right)\right] ;\left[\mathrm{A}\left(x_{s}\right) ; \mathrm{A}\left(x_{r}\right)\right]\right]
$$

$$
\begin{aligned}
\langle 0|\left[\left[\left[\mathrm{A}\left(-x_{i}\right) ; \mathrm{A}\left(-x_{j}\right)\right] ;\right.\right. & \left.\left.\mathrm{A}\left(-x_{s}\right)\right] ; \mathrm{A}\left(-x_{r}\right)\right]|0\rangle \\
& =-\left\langle 0\left|\left[\left[\left[\mathrm{~A}\left(x_{i}\right) ; \mathrm{A}\left(x_{j}\right)\right] ; \mathrm{A}\left(x_{s}\right)\right] ; \mathrm{A}\left(x_{r}\right)\right]\right| 0\right\rangle
\end{aligned}
$$

verschwindet der zweite Term in Gleichung (60).

Also gilt:

$$
I(k)=-\frac{2 i}{(2 \pi)^{10}} \int d^{4} x e^{i k x}\left\langle 0\left|\left[R\left(x_{i} \mid x_{m}\right) ; R\left(x_{j} \mid x_{n}\right)\right]\right| 0\right\rangle .
$$

Fügen wir zwischen die beiden auftretenden retardierten Kommutatoren ein vollständiges System von Eigenzuständen zu $P^{2}$ und $P^{(\mu)}$ ein:

mit

$|0\rangle\left\langle 0\left|+\int d \varrho\left(M^{2}\right) \int d^{4} p \theta(p) \delta\left(p^{2}-m^{2}\right)\right| p ; M\right\rangle\langle M ; p|=1$

$$
\left\{\begin{array}{l}
P^{(\mu)}|p ; M\rangle=p^{(\mu)}|p ; M\rangle ; P^{2}|p ; M\rangle=M^{2}|p ; M\rangle \\
d \delta\left(M^{2}\right)=\delta\left(m^{2}-M^{2}\right) d M^{2}+d \bar{\varrho}\left(M^{2}\right) \\
\text { Träger von } d \bar{\varrho}\left(M^{2}\right)>4 m^{2}
\end{array}\right\}
$$

so erhalten wir schließlich, da für $\left(k_{j}+k_{n}\right)^{2}<4 m^{2}$ nur der diskrete Anteil $\delta\left(m^{2}-M^{2}\right)$ des Maßes $d \varrho$ einen Beitrag liefert:

$$
\begin{aligned}
I(k) & =2 i \int d^{4} p \theta(p) \delta\left(p^{2}-m^{2}\right) \times \\
& \times\left\{\left\langle 0\left|\widetilde{R}\left(k_{i} \mid k_{m}\right)\right| p ; m\right\rangle\left\langle m ; p\left|\widetilde{R}\left(k_{j} \mid k_{n}\right)\right| 0\right\rangle\right. \\
& \left.-\left\langle 0\left|\widetilde{R}\left(k_{j} \mid k_{n}\right)\right| p ; m\right\rangle\left\langle m ; p\left|\widetilde{R}\left(k_{i} \mid k_{m}\right)\right| 0\right\rangle\right\} .
\end{aligned}
$$

Andererseits folgt mit

$$
\tilde{r}\left(k_{j} \mid k_{i} ; p\right)-\tilde{a}\left(k_{i} \mid k_{j} ; p\right)=-i\left\langle 0\left|\left[\widetilde{R}\left(k_{j} \mid k_{i}\right) ; \tilde{A}(p)\right]\right| 0\right\rangle
$$

sowie Folgerung $\mathrm{FI}_{1}$ Gleichung (32):

$$
\begin{aligned}
A(k)=I(k) & -4 \pi i \int d^{4} p\left\{\left\langle 0\left|\widetilde{R}\left(k_{j} \mid k_{n}\right) \tilde{\mathrm{A}}(-p)\right| 0\right\rangle-\right. \\
& \left.-\left\langle 0\left|\tilde{\mathrm{A}}(-p) \widetilde{R}\left(k_{j} / k_{n}\right)\right| 0\right\rangle\right\}\left(p^{2}-m^{2}\right) \tilde{r}\left(k_{i} \mid k_{m}, p\right) .
\end{aligned}
$$


Für $\left(k_{j}+k_{n}\right)^{2}<4 m^{2}$ ergibt sich daraus mit der Spektrumsbedingung (AII) bzw. (WII'):

$$
\begin{aligned}
A(k) & =I(k)-4 \pi i \int d^{4} p \theta(p) \delta\left(p^{2}-m^{2}\right) \times \\
& \times\left\{\left\langle 0\left|\widetilde{R}\left(k_{j} \mid k_{n}\right)\right| p, m\right\rangle\left(p^{2}-m^{2}\right) \tilde{r}\left(k_{i} \mid k_{m}, p\right)-\right. \\
& \left.-\left\langle m ; p\left|\widetilde{R}\left(k_{j} \mid k_{n}\right)\right| 0\right\rangle\left(p^{2}-m^{2}\right) \tilde{r}\left(k_{i} \mid k_{m},-p\right)\right\} .
\end{aligned}
$$

Es sei nun $\left\{f_{\alpha}(x)\right\}$ ein vollständiges System positiv frequenter Lösungen der Klein-Gordon-Gleichung zur Masse $m$. Aus der unter den Voraussetzungen (AI) - (AIV) bzw. (WI) - (WIII) gültigen schwachen $Z u$ standskonvergenz auf den asymptotischen Hilberträumen $\mathfrak{F}^{\mathrm{ex}}[19]$, die den Einteilchen-Unterraum aus $\mathfrak{S}$ enthalten:

mit

$$
w-\lim _{t \rightarrow \pm \infty} A^{f} \alpha(t)^{*}|0\rangle=\left(\begin{array}{c}
A_{\text {out }}^{f} \\
\text { in }
\end{array}\right)^{*}|0\rangle
$$

$$
\mathrm{A}_{(\mathrm{ex})}^{f_{\alpha}}(t)=: i \int_{t=x^{(0)}} d^{3} x \overline{f_{\alpha}(x)} \frac{\overleftrightarrow{\partial}}{\partial x^{(0)}} \mathrm{A}(x)_{(\mathrm{ex})}
$$

folgt mit den üblichen Reduktionsverfahren [20], [37]:

$$
\begin{aligned}
\left(k^{2}-m^{2}\right) \tilde{r}(p / q,-k) & =\langle 0|\widetilde{R}(p / q)| k ; m\rangle(2 \pi)^{-1} \\
\left(k^{2}-m^{2}\right) \tilde{r}(p / q, k) & =\langle m ; k|\widetilde{R}(p / q)| 0\rangle(2 \pi)^{-1} \\
\text { für } \quad k^{(0)} & =+\sqrt{\vec{k}^{2}+m^{2} .}
\end{aligned}
$$

Damit erhalten wir aus Gleichung (68):

$$
\begin{aligned}
A(k) & =I(k)+2 i \int d^{4} p \theta(p) \delta\left(p^{2}-m^{2}\right) \times \\
& \times\left\{\left\langle 0\left|\widetilde{R}\left(k_{i} \mid k_{m}\right)\right| p ; m\right\rangle\left\langle m ; p\left|\widetilde{R}\left(k_{j} \mid k_{n}\right)\right| 0\right\rangle-\right. \\
& \left.-\left\langle 0\left|\widetilde{R}\left(k_{j} \mid k_{n}\right)\right| p ; m\right\rangle\left\langle m ; p\left|\widetilde{R}\left(k_{i} \mid k_{m}\right)\right| 0\right\rangle\right\} .
\end{aligned}
$$

Durch Vergleich mit Gleichung (65) erhalten wir:

$$
A(k)=0 \text { für } \quad\left(k_{j}+k_{n}\right)^{2}<4 m^{2}
$$

und daraus mit der Steinmann-Relation:

$$
\tilde{r}_{i j}(k)^{\mathrm{I}}-\tilde{a}_{m n}(k)^{\mathrm{I}}=0 \text { für }\left(k_{j}+k_{n}\right)^{2}<4 m^{2} .
$$

Es sei nun $A_{j n}\left(\varepsilon / 4 m^{2}\right)$ eine komplexe Umgebung des Abschnittes $\left(k_{j}+k_{n}\right)^{2}<4 m^{2}$ der reellen $k$-Achse.

$A_{j n}\left(\varepsilon / 4 m^{2}\right)=:\left\{p: \sum_{r=1}^{4} p_{r}=0 ;|q|<\varepsilon ;\left(k_{j}+k_{n}\right)^{2}<4 m^{2} ; \varepsilon>0\right\}$.

Dann ist $\tilde{r}_{i j}^{\prime}(p)^{\mathrm{I}}$ regulär für $p \in \mathfrak{S}_{i j}^{n-}$ mit

$$
\mathfrak{D}_{i j}^{n-}=: A_{j n}\left(\varepsilon / 4 m^{2}\right) \cap T_{i j}^{-}
$$

und $\tilde{a}_{m n}^{\prime}(p)^{\mathrm{I}}$ regulär für $p \in \mathfrak{D}_{m n}^{j+}$ mit

$$
\mathfrak{S}_{m n}^{j+}=: A_{j n}\left(\varepsilon / 4 m^{2}\right) \cap T_{m n}^{+} .
$$


Da sich $T_{i j}^{-}$und $T_{m n}^{+}$nur im Vorzeichen von $\left(q_{j}^{(0)}+q_{n}^{(0)}\right)$ unterscheiden, so folgt mit den Gleichungen (73)-(76) aus dem verallgemeinerten Edge-of-the-Wedge-Theorem [8], [36], daß $\tilde{r}_{i j}^{\prime}(p)^{\mathrm{I}}$ und $\tilde{a}_{m n}^{\prime}(p)^{\mathrm{I}}$ eine gemeinsame analytische Fortsetzung in den Durchschnitt $B_{i j}^{m n}$ von $A_{j n}\left(\varepsilon / 4 m^{2}\right)$ und der abgeschlossenen konvexen Hülle von $\left\{T_{i j}^{-} \cup T_{m n}^{+}\right\}$ haben:

$$
B_{i j}^{m n}=: A_{j n}\left(\varepsilon / 4 m^{2}\right) \cap \overline{\operatorname{conv}\left\{T_{i j}^{-} \cup T_{m n}^{+}\right\}} .
$$

Die Holomorphie-Envelope von $T_{i j}^{-} \cup T_{m n}^{+} \cup B_{i j}^{m n}$ ist aber gerade wieder durch $\sum_{i j}^{m} n\left(4 m^{2}\right)$ gegeben [28].

Schließlich ist noch die analytische Fortsetzbarkeit in die reellen Punkte $B\left(m^{2} ; 4 m^{2}\right)$ zu zeigen. $B\left(m^{2} ; 4 m^{2}\right)$ ist der Durchschnitt der sieben reellen Bereiche

$$
\begin{aligned}
H_{j} & =:\left\{k: \sum_{r=1}^{4} k_{r}=0 ; k_{j}^{2}<m^{2}\right\} \quad j=1 ; 2 ; 3 ; 4 \\
H_{i n} & =:\left\{k: \sum_{r=1}^{4} k_{r}=0 ;\left(k_{i}+k_{n}\right)^{2}<4 m^{2}\right\} \quad i \neq n, 1 \leqq i ; n \leqq 4 .
\end{aligned}
$$

In diesen Bereichen sind jeweils zwei der zweiunddreißig $r(k)^{\mathrm{I}}$-Funktionen infolge von (50) und (51) gleich, so daß in $B\left(m^{2} ; 4 m^{2}\right)$ alle zweiunddreißig Funktionen übereinstimmen. Wenden wir nun das Edge-of-the-WedgeTheorem auf zwei Funktionen, z. B. $\left\{\tilde{r}_{i}^{\prime}(p)^{\mathrm{I}} ; \tilde{a}_{i}^{\prime}(p)^{\mathrm{I}}\right\}$ oder $\left\{\tilde{r}_{i j}^{\prime}(p)^{\mathrm{I}} ; \tilde{a}_{i j}^{\prime}(p)^{\mathrm{I}}\right\}$ an, für die die zugehörigen Röhren $\left\{T_{i}^{-} ; T_{i}^{+}\right\}$bzw. $\left\{T_{i j}^{-} ; T_{i j}^{+}\right\}$durch Spiegelung am Ursprung auseinander hervorgehen, so folgt die Behauptung.

\section{Rekonstruktion der Wightman-Funktion}

Nach Jost [30] und Steinmans [26] gibt es zu gegebenem $g(p)$ $[G(p)]$ mit den Eigenschaften aus Satz I [Satz II] eindeutige Funktionen $K_{3}\left(x_{1} ; x_{2} ; x_{3}\right) \quad\left[K_{4}\left(x_{1} ; x_{2} ; x_{3} ; x_{4}\right)\right]$ mit den folgenden Eigenschaften $(\mathrm{KI})-(\mathrm{KIV})$ der Art, daß die gemäß

$$
\begin{gathered}
r\left(x / x_{1} ; x_{2}\right)=-\theta\left(x-x_{1}\right) \theta\left(x_{1}-x_{2}\right) K_{3}\left(x, x_{1}, x_{2}\right)- \\
-\theta\left(x-x_{2}\right) \theta\left(x_{2}-x_{1}\right) K_{3}\left(x, x_{2}, x_{1}\right) \\
r\left(x / x_{1} ; x_{2} ; x_{3}\right)=(-i)^{3} \sum_{\text {Perm. }\{1 ; 2,3\}} \theta\left(x-x_{1}\right) \theta\left(x_{1}-x_{2}\right) \theta\left(x_{2}-x_{3}\right) \times \\
\times K_{4}\left(x, x_{1}, x_{2}, x_{3}\right)
\end{gathered}
$$

berechneten $r$-Funktionen die vorgegebenen sind:

(KI) Lorentzinvarianz:

$$
\underset{4}{K_{3}}(x)=\underset{4}{K_{3}}(\Lambda x+a) \text { für } \quad\left(\Lambda_{i} a\right) \in i L g_{+}^{\nearrow} .
$$


(KII) Spektrumsbedingung:

$$
\begin{array}{rlll}
\widetilde{K}_{3}\left(k_{1}, k_{2}, k_{3}\right)=0 & \text { für } \sum_{j=1}^{3} k_{j} \neq 0 & \text { oder } & \left(k_{1}+k_{2}\right)^{2}<m^{2} \\
& \text { oder }\left\{k_{1}^{2}<m^{2}\right. & \text { und } & \left.k_{2}^{2}<m^{2}\right\}
\end{array}
$$

$\widetilde{K}_{4}\left(k_{1}, k_{2}, k_{3}, k_{4}\right)=0$ für $\sum_{r=1}^{4} k_{r} \neq 0$ oder $k_{4}^{2}<m^{2}$

$\widetilde{K}_{4}\left(k_{1}, k_{2}, k_{3}, k_{4}\right)-\widetilde{K}_{4}\left(k_{1}, k_{2}, k_{4}, k_{3}\right)=0$ für $\left(k_{3}+k_{4}\right)^{2}<m^{2}$.

(KIII) Lokalität:

$$
\begin{aligned}
& K_{3}\left(x_{1}, x_{2}, x_{3}\right)=0 \text { für } \quad\left(x_{1}-x_{2}\right)^{2}<0 \\
& \text { oder }\left\{\left(x_{2}-x_{3}\right)^{2}<0 \text { und }\left(x_{1}-x_{3}\right)^{2}<0\right\} \\
& K_{4}\left(x_{1}, x_{2}, x_{3}, x_{4}\right)=0 \text { für }\left(x_{1}-x_{2}\right)^{2}<0 . \\
& \text { (KIV) (Jakobi-)Identitäten: }
\end{aligned}
$$

$$
\begin{gathered}
K_{3}\left(x_{1}, x_{2}, x_{3}\right)+K_{3}\left(x_{2}, x_{1}, x_{3}\right)=0 \\
K_{3}\left(x_{1}, x_{2}, x_{3}\right)+K_{3}\left(x_{2}, x_{3}, x_{1}\right)+K_{3}\left(x_{3}, x_{1}, x_{2}\right)=0 \\
K_{4}\left(x_{1}, x_{2}, x_{3}, x_{4}\right)+K_{4}\left(x_{2}, x_{1}, x_{3}, x_{4}\right)=0 \\
K_{4}\left(x_{1}, x_{2}, x_{3}, x_{4}\right)+K_{4}\left(x_{2}, x_{3}, x_{1}, x_{4}\right)+K_{4}\left(x_{3}, x_{1}, x_{2}, x_{4}\right)=0 \\
K_{4}\left(x_{1}, x_{2}, x_{3}, x_{4}\right)-K_{4}\left(x_{1}, x_{2}, x_{4}, x_{3}\right) \\
=K_{4}\left(x_{3}, x_{4}, x_{2}, x_{1}\right)-K_{4}\left(x_{3}, x_{4}, x_{1}, x_{2}\right) .
\end{gathered}
$$

Die $K$-Funktionen sind explizite gegeben durch:

$$
\begin{gathered}
K_{3}\left(x_{1}, x_{2}, x_{3}\right)=r\left(x_{2} / x_{1}, x_{3}\right)-a\left(x_{1} / x_{2}, x_{3}\right)- \\
-r\left(x_{1} / x_{2} ; x_{3}\right)+a\left(x_{2} / x_{1}, x_{3}\right) \\
K_{4}\left(x_{1}, x_{2}, x_{3}, x_{4}\right)=-S_{14}(x)+S_{14}(-x)+S_{24}(x)-S_{24}(-x) \\
\text { mit } S_{n j}(x)=i\left[r_{n}(x)-r_{n j}(x)\right] .
\end{gathered}
$$

Entsprechend den Gleichungen (46) und (47) spalten wir die $K_{4}$-Funktion in zwei Anteile auf:

$$
\begin{aligned}
& K_{4}\left(x_{1}, x_{2}, x_{3}, x_{4}\right)^{\mathrm{II}}=:-S_{14}(x)^{\mathrm{II}}+S_{14}(-x)^{\mathrm{II}}+S_{24}(x)^{\mathrm{II}}-S_{24}(-x)^{\mathrm{II}} \\
& \text { mit } S_{l j}(x)^{\mathrm{II}}=+i\left\{r_{l}(x)^{\mathrm{I}}-r_{l j}(x)^{\mathrm{I}}\right\} \\
& K_{4}\left(x_{1}, x_{2}, x_{3}, x_{4}\right)^{\mathrm{I}}=: K_{4}\left(x_{1}, x_{2}, x_{3}, x_{4}\right)-K_{4}\left(x_{1}, x_{2}, x_{3}, x_{4}\right)^{\mathrm{II}}
\end{aligned}
$$

Jede dieser beiden Funktionen erfüllt für sich die Bedingungen (KI) bis (KIV). Außerdem hat aber $K_{4}(x)^{\mathrm{II}}$ den kleineren Träger:

$\left(\mathrm{KII}^{\prime}\right): \widetilde{K}_{4}\left(k_{1} ; k_{2} ; k_{3} ; k_{4}\right)^{\mathrm{II}}-\widetilde{K}_{4}\left(k_{1} ; k_{2} ; k_{4} ; k_{3}\right)^{\mathrm{II}}=0$ für $\left(k_{3}+k_{4}\right)^{2}<$ $<4 m^{2}$. 
Über die Einteilchensingularitäten in Relativistischen Quantenfeldtheorien 193

Daß $K_{4}(x)^{\mathrm{II}}$ diese Eigenschaften hat, ergibt sich unmittelbar aus Satz III, wenn man noch die aus Gleichung (83) mit der Symmetrie der $r_{j}(x)$ folgende Relation

$$
\begin{aligned}
K_{4}\left(x_{1}, x_{2}, x_{3}, x_{4}\right)^{\mathrm{II}} & -K_{4}\left(x_{1}, x_{2}, x_{4}, x_{3}\right)^{\mathrm{II}} \\
& =i\left\{\left[r_{14}(x)^{\mathrm{I}}-a_{23}(x)^{\mathrm{I}}\right]+\left[r_{23}(x)^{\mathrm{I}}-a_{14}(x)^{\mathrm{I}}\right]\right. \\
& \left.-\left[r_{13}(x)^{\mathrm{I}}-a_{24}(x)^{\mathrm{I}}\right]-\left[r_{24}(x)^{\mathrm{I}}-a_{13}(x)^{\mathrm{I}}\right]\right\}
\end{aligned}
$$

beachtet.

Aus den Definitionsgleichungen (83) und (84) folgt durch Einsetzen von (46) und (47) mit Gleichung (81) sowie

$$
\begin{aligned}
& \left\langle 0\left|\left[\widetilde{R}\left(k_{j} \mid k_{r}\right) ; \tilde{\mathrm{A}}(k)\right]\right| 0\right\rangle \\
& =\frac{1}{m^{2}-\left(\begin{array}{c}
\left.k_{j}+i \varepsilon\right)^{2} \\
r^{2}
\end{array}\right.}\left(m^{2}-k_{j}^{2}\right)\left\langle 0\left|\left[\widetilde{R}\left(k_{j} \mid k\right) ; \tilde{\mathrm{A}}(k)\right]\right| 0\right\rangle
\end{aligned}
$$

die Darstellung für $K_{4}(k)^{\mathrm{I}}$ :

$$
\begin{aligned}
& \widetilde{K}_{4}\left(k_{1}, k_{2}, k_{3}, k_{4}\right)^{\mathrm{I}}=-2 \pi \int d^{4} p \times \\
& \times\left\{\widetilde{K}_{3}\left(k_{1},-p, k_{r}\right) \overleftrightarrow{\Delta}_{\text {ret }}(p)\left\langle 0\left|\left[\widetilde{R}\left(p / k_{2}\right) ; \widetilde{\mathrm{A}}\left(k_{4}\right)\right]\right| 0\right\rangle+\right. \\
& +\widetilde{K}_{3}\left(-p, k_{2}, k_{4}\right) \overleftrightarrow{\Delta}_{\mathrm{ret}}(p)\left\langle 0\left|\left[\widetilde{R}\left(p / k_{1}\right) ; \widetilde{\mathrm{A}}\left(k_{3}\right)\right]\right| 0\right\rangle+ \\
& +\widetilde{K}_{3}\left(k_{1},-p, k_{4}\right) \overleftrightarrow{\Delta}_{\mathrm{ret}}(p)\left\langle 0\left|\left[\widetilde{R}\left(p / k_{2}\right) ; \widetilde{\mathrm{A}}\left(k_{3}\right)\right]\right| 0\right\rangle+ \\
& +\widetilde{K}_{3}\left(-p, k_{2}, k_{3}\right) \overleftrightarrow{\Delta}_{\text {ret }}(p)\left\langle 0\left|\left[\widetilde{R}\left(p \mid k_{1}\right) ; \widetilde{\mathrm{A}}\left(k_{4}\right)\right]\right| 0\right\rangle+ \\
& +\widetilde{K}_{3}\left(k_{1},-p, k_{2}\right) \overleftrightarrow{\Delta}_{\text {ret }}(p)\left\langle 0\left|\left[\widetilde{R}\left(p / k_{3}\right) ; \widetilde{A}\left(k_{4}\right)\right]\right| 0\right\rangle+ \\
& +\widetilde{K}_{3}\left(-p, k_{3}, k_{4}\right) \overleftrightarrow{\Delta_{\mathrm{ret}}}(p)\left\langle 0\left|\left[\widetilde{R}\left(p / k_{1}\right) ; \widetilde{\mathrm{A}}\left(k_{2}\right)\right]\right| 0\right\rangle+ \\
& +\widetilde{K}_{3}\left(k_{3},-p, k_{4}\right) \overleftrightarrow{\Delta}_{\text {ret }}(p)\left\langle 0\left|\left[\widetilde{R}\left(p \mid k_{2}\right) ; \widetilde{A}\left(k_{1}\right)\right]\right| 0\right\rangle+ \\
& +\widetilde{K}_{3}\left(-p, k_{2}, k_{1}\right) \overleftrightarrow{\Delta}_{\mathrm{ret}}(p)\left\langle 0\left|\left[\widetilde{R}\left(p / k_{3}\right) ; \widetilde{\mathrm{A}}\left(k_{4}\right)\right]\right| 0\right\rangle- \\
& -i\left\langle 0\left|\left[\widetilde{R}\left(-p / k_{1}\right) ; \widetilde{\mathrm{A}}\left(k_{3}\right)\right]\right| 0\right\rangle \overleftrightarrow{\Delta}(p)\left\langle 0\left|\left[\widetilde{R}\left(p / k_{2}\right) ; \widetilde{\mathrm{A}}\left(k_{4}\right)\right]\right| 0\right\rangle- \\
& -i\left\langle 0\left|\left[\widetilde{R}\left(-p / k_{1}\right) ; \widetilde{\mathrm{A}}\left(k_{4}\right)\right]\right| 0\right\rangle \overleftrightarrow{\Delta}(p)\left\langle 0\left|\left[\widetilde{R}\left(p / k_{2}\right) ; \widetilde{\mathrm{A}}\left(k_{3}\right)\right]\right| 0\right\rangle- \\
& -i\left\langle 0\left|\left[\widetilde{R}\left(-p / k_{1}\right) ; \widetilde{\mathrm{A}}\left(k_{2}\right)\right]\right| 0\right\rangle \overleftrightarrow{\Delta}(p)\left\langle 0\left|\left[\widetilde{R}\left(p / k_{3}\right) ; \widetilde{\mathrm{A}}\left(k_{4}\right)\right]\right| 0\right\rangle- \\
& \left.-i\left\langle 0\left|\left[\widetilde{R}\left(-p / k_{3}\right) ; \widetilde{\mathrm{A}}\left(k_{4}\right)\right]\right| 0\right\rangle \overleftrightarrow{\Delta}(p)\left\langle 0\left|\left[\widetilde{R}\left(p / k_{2}\right) ; \widetilde{\mathrm{A}}\left(k_{1}\right)\right]\right| 0\right\rangle\right\}
\end{aligned}
$$

mit

$$
\begin{aligned}
\overleftrightarrow{\Delta}_{\mathrm{ret}} & (p)=\overleftarrow{\left(m^{2}-p^{2}\right)} \frac{1}{m^{2}-(k+i \varepsilon)^{2}} \overline{\left(m^{2}-p^{2}\right)} \\
\overleftrightarrow{\Delta}(p) & =-2 \pi i \overleftarrow{\left(m^{2}-p^{2}\right)} \varepsilon\left(p^{0}\right) \delta\left(p^{2}-m^{2}\right) \overline{\left(m^{2}-p^{2}\right)}
\end{aligned}
$$

Schließlich existieren für jedes gegebene $K_{i}(x)(i=3,4)$ mit den Eigenschaften $(\mathrm{KI})-(\mathrm{KIV})$ eindeutig trunkierte Wightman-Funktionen, d. h. Funktionen mit den Eigenschaften (WI), (WII') und (WIII), der 
Art, daß die daraus berechneten dreifachen bzw. vierfachen Kommutatoren die vorgegebenen $K$-Funktionen sind [21]; [25]. Explizite sind die trunkierten Wightman-Funktionen gegeben durch:

$$
\begin{aligned}
\widetilde{W}\left(k_{1}, k_{2}, k_{3}\right)^{T} & =\widetilde{W}\left(k_{1}, k_{2}, k_{3}\right)=\theta\left(k_{1}+k_{2}\right) \theta\left(-k_{2}\right) \widetilde{K}_{3}\left(k_{1}, k_{2}, k_{3}\right)+ \\
& +\theta\left(k_{1}\right) \theta\left(k_{2}\right) \widetilde{K}_{3}\left(k_{3}, k_{2}, k_{1}\right) \widetilde{W}\left(k_{1}, k_{2}, k_{3}, k_{4}\right)^{T} \\
& =\theta\left(k_{1}\right)\left\{\theta\left(k_{1}+k_{2}\right) \times\right. \\
& \times\left[\theta\left(k_{1}+k_{2}+k_{3}\right) \widetilde{K}_{4}\left(k_{1}, k_{2}, k_{3}, k_{4}\right)-\right. \\
& \left.-\theta\left(k_{3}\right) \widetilde{K}_{4}\left(k_{1}, k_{2}, k_{4}, k_{3}\right)\right]-\theta\left(k_{1}+k_{3}\right) \theta\left(k_{2}\right) \times \\
& \times \widetilde{K}_{4}\left(k_{1}, k_{3}, k_{4}, k_{2}\right)-\theta\left(k_{2}+k_{4}\right) \theta\left(k_{1}+k_{2}+k_{3}\right) \times \\
& \times \widetilde{K}_{4}\left(k_{1}, k_{3}, k_{2}, k_{4}\right)+\theta\left(k_{2}+k_{3}\right)\left[\theta\left(k_{2}\right) \times\right. \\
& \left.\left.\times \widetilde{K}_{4}\left(k_{1}, k_{4}, k_{3}, k_{2}\right)-\theta\left(-k_{3}\right) \widetilde{K}_{4}\left(k_{1}, k_{4}, k_{2}, k_{3}\right)\right]\right\} .
\end{aligned}
$$

Entsprechend der Gleichung (84) spalten wir die $W$-Funktionen wiederum in zwei Anteile auf. Wir definieren zunächst $W^{\mathrm{I}}\left(k_{1}, \ldots k_{4}\right)$ durch die zu (89) analoge Gleichung, die man erhält, wenn man auf der rechten Seite $K_{4}(k)$ durch $K_{4}(k)^{\mathrm{I}}$ ersetzt. Aus der Darstellung (87) für $K_{4}(k)^{\mathrm{I}}$ folgt dann nach längeren Rechnungen unter Benutzung von

$$
\begin{aligned}
\widetilde{K}_{3}\left(k_{1}, k_{2}, k_{3}\right) & =\widetilde{W}\left(k_{1}, k_{2}, k_{3}\right)-\widetilde{W}\left(k_{2}, k_{1}, k_{3}\right)+ \\
& +\widetilde{W}\left(k_{3}, k_{2}, k_{1}\right)-\widetilde{W}\left(k_{3}, k_{1}, k_{2}\right)
\end{aligned}
$$

sowie den Trägereigenschaften $\left(\mathrm{WII}^{\prime}\right)$ von $\widetilde{W}\left(k_{1}, k_{2}, k_{3}\right)$ : die Darstellung (91) für $W^{\mathrm{I}}$.

Aus der Darstellung (91) folgt sofort, daß $W^{\mathrm{I}}$ lorentzinvariant ist, und mit den Trägereigenschaften der auftretenden Dreipunktfunktionen die Spektrumseigenschaft $\left(\mathrm{WII}^{\prime}\right)$.

$$
\begin{aligned}
\widetilde{W}^{\mathrm{I}}\left(k_{1}, k_{2}, k_{3}, k_{4}\right) & =-2 \pi \int d^{4} p \times \\
& \times\left\{\widetilde { W } ( k _ { 1 } , k _ { 2 } , - p ) \overleftrightarrow { \Delta } _ { \mathrm { ret } } ( p ) \left[\left\langle 0\left|\widetilde{R}\left(p / k_{3}\right) \tilde{\mathrm{A}}\left(k_{4}\right)\right| 0\right\rangle+\right.\right. \\
& +\left\langle 0\left|\tilde{\mathrm{A}}\left(k_{3}\right) \widetilde{R}\left(p / k_{4}\right)\right| 0\right\rangle+ \\
& +\widetilde{W}\left(-p, k_{3}, k_{4}\right) \overleftrightarrow{\Delta}_{\mathrm{ret}}(p)\left[\left\langle 0\left|\widetilde{R}\left(p / k_{1}\right) \tilde{\mathrm{A}}\left(k_{2}\right)\right| 0\right\rangle+\right. \\
& +\left\langle 0\left|\tilde{\mathrm{A}}\left(k_{1}\right) \widetilde{R}\left(p / k_{2}\right)\right| 0\right\rangle+ \\
& +\widetilde{W}\left(k_{1},-p, k_{4}\right) \overleftrightarrow{\Delta}_{\mathrm{ret}}(p)\left[\left\langle 0\left|\tilde{\mathrm{A}}\left(k_{2}\right) \widetilde{R}\left(p / k_{3}\right)\right| 0\right\rangle+\right. \\
& +\left\langle 0\left|\widetilde{R}\left(p / k_{2}\right) \tilde{\mathrm{A}}\left(k_{3}\right)\right| 0\right\rangle+ \\
& +\widetilde{W}\left(k_{1}, k_{3},-p\right) \overleftrightarrow{\Delta}_{\mathrm{ret}}(p)\left\langle 0\left|\tilde{\mathrm{A}}\left(k_{2}\right) \widetilde{R}\left(p / k_{4}\right)\right| 0\right\rangle+ \\
& +\widetilde{W}\left(k_{1},-p, k_{3}\right) \overleftrightarrow{\Delta}_{\mathrm{ret}}(p)\left\langle 0\left|\widetilde{R}\left(p / k_{2}\right) \tilde{\mathrm{A}}\left(k_{4}\right)\right| 0\right\rangle+ \\
& +\widetilde{W}\left(k_{2}, k_{3},-p\right) \overleftrightarrow{\Delta}_{\mathrm{ret}}(p)\left\langle 0\left|\tilde{\mathrm{A}}\left(k_{1}\right) \widetilde{R}\left(p / k_{4}\right)\right| 0\right\rangle+
\end{aligned}
$$




$$
\begin{aligned}
& +\widetilde{W}\left(-p, k_{2}, k_{3}\right) \overleftrightarrow{\Delta}_{\mathrm{ret}}(p)\left\langle 0\left|\widetilde{R}\left(p / k_{1}\right) \tilde{\mathrm{A}}\left(k_{4}\right)\right| 0\right\rangle+ \\
& +\widetilde{W}\left(-p, k_{2}, k_{4}\right) \overleftrightarrow{\Delta}_{\mathrm{ret}}(p)\left\langle 0\left|\widetilde{R}\left(p / k_{1}\right) \tilde{\mathrm{A}}\left(k_{3}\right)\right| 0\right\rangle+ \\
& +\widetilde{W}\left(k_{2},-p, k_{4}\right) \overleftrightarrow{\Delta}_{\mathrm{ret}}(p)\left\langle 0\left|\tilde{\mathrm{A}}\left(k_{1}\right) \widetilde{R}\left(p / k_{3}\right)\right| 0\right\rangle- \\
& -i\left[\left\langle 0\left|\tilde{\mathrm{A}}\left(k_{1}\right) \widetilde{R}\left(-p \mid k_{2}\right)\right| 0\right\rangle+\right. \\
& +\left\langle 0\left|\widetilde{R}\left(-p / k_{1}\right) \tilde{\mathrm{A}}\left(k_{2}\right)\right| 0\right\rangle \overleftrightarrow{\Delta}_{+}(p) \times \\
& \times\left[\left\langle 0\left|\tilde{\mathrm{A}}\left(k_{3}\right) \widetilde{R}\left(p / k_{4}\right)\right| 0\right\rangle+\left\langle 0\left|\widetilde{R}\left(p / k_{3}\right) \tilde{\mathrm{A}}\left(k_{4}\right)\right| 0\right\rangle\right]- \\
& -i\left\langle 0\left|\widetilde{R}\left(-p / k_{1}\right) \tilde{\mathrm{A}}\left(k_{4}\right)\right| 0\right\rangle \overleftrightarrow{\Delta}_{+}(p) \times \\
& \times\left[\left\langle 0\left|\tilde{\mathrm{A}}\left(k_{2}\right) \widetilde{R}\left(p / k_{3}\right)\right| 0\right\rangle+\left\langle 0\left|\widetilde{R}\left(p / k_{2}\right) \tilde{\mathrm{A}}\left(k_{3}\right)\right| 0\right\rangle\right]- \\
& -i\left\langle 0\left|\tilde{\mathrm{A}}\left(k_{1}\right) \widetilde{R}\left(p / k_{4}\right)\right| 0\right\rangle \overleftrightarrow{\Delta_{+}}(p) \times \\
& \times\left[\left\langle 0\left|\tilde{\mathrm{A}}\left(k_{2}\right) \widetilde{R}\left(-p \mid k_{3}\right)\right| 0\right\rangle+\left\langle 0\left|\widetilde{R}\left(-p / k_{2}\right) \tilde{\mathrm{A}}\left(k_{3}\right)\right| 0\right\rangle\right]- \\
& -i\left\langle 0\left|\widetilde{R}\left(-p / k_{1}\right) \tilde{\mathrm{A}}\left(k_{3}\right)\right| 0\right\rangle \overleftrightarrow{\Delta}_{+}(p) \times \\
& \times\left[\left\langle 0\left|\tilde{\mathrm{A}}\left(k_{2}\right) \widetilde{R}\left(p / k_{4}\right)\right| 0\right\rangle+\left\langle\left|\widetilde{R}\left(p / k_{2}\right) \tilde{\mathrm{A}}\left(k_{4}\right)\right| 0\right\rangle\right]- \\
& -i\left\langle 0\left|\tilde{\mathrm{A}}\left(k_{1}\right) \widetilde{R}\left(p / k_{3}\right)\right| 0\right\rangle \times \\
& \times\left[\overleftrightarrow{\Delta_{+}}(p)\left\langle 0\left|\widetilde{R}\left(-p / k_{2}\right) \tilde{\mathrm{A}}\left(k_{4}\right)\right| 0\right\rangle+\right. \\
& \left.\left.+\overleftrightarrow{\Delta_{+}}(-p)\left\langle 0\left|\tilde{\mathrm{A}}\left(k_{2}\right) \widetilde{R}\left(-p / k_{4}\right)\right| 0\right\rangle\right]\right\}
\end{aligned}
$$

mit

$$
\overleftrightarrow{\Delta}_{+}(p)=-2 \pi i \overleftarrow{\left(m^{2}-p^{2}\right)} \theta\left(p^{0}\right) \delta\left(p^{2}-m^{2}\right) \overline{\left(m^{2}-p^{2}\right)}
$$

$\widetilde{W}^{\mathrm{II}}\left(k_{1}, \ldots, k_{4}\right)$ wird dann definiert durch :

$$
\widetilde{W}_{\mathrm{II}}\left(k_{1}, k_{2}, k_{3}, k_{4}\right)=: \widetilde{W}^{T}\left(k_{1}, k_{2}, k_{3}, k_{4}\right)-\widetilde{W}^{\mathrm{I}}\left(k_{1}, k_{2}, k_{3}, k_{4}\right) .
$$

Die Lokalität von $W^{\mathrm{II}}(x)$ folgt natürlich unmittelbar mit den Überlegungen von O. STEINMANN [26] aus der Gleichung (89) analogen Darstellung durch $K_{4}(x)^{\mathrm{I}}$-Funktionen. Ohne Benutzung der retardierten Drei- bzw. Vierpunktfunktionen folgt die Lokalität zunächst für $W^{\mathrm{I}}\left(x_{1}, \ldots, x_{4}\right)$ direkt aus der fouriertransformierten Darstellung (91), und damit auch aus Gleichung (93) für $W^{\mathrm{II}}\left(x_{1}, \ldots, x_{4}\right)$.

Die Gültigkeit von (WII") kann ebenfalls mit der erwähnten Darstellung durch $K_{4}(x)^{\mathrm{I}}$-Funktionen mit einigen Modifikationen des Steinmannschen Rekonstruktionsbeweises [26] gezeigt werden. Für die weiteren Überlegungen ist es aber sinnvoll, einen direkten Beweis mit Hilfe der Gleichung (91) anzugeben.

Infolge der Trägerbedingung (WII') gilt $k_{2} \notin V_{+}^{m}$ und $k_{3} \notin V^{m}$ für $0<\left(k_{1}+k_{2}\right)^{2}<4 m^{2}$. Für diesen Variabilitätsbereich verschwinden 
aber alle Terme der Gleichung (91) bis auf die folgenden vier:

$$
\begin{aligned}
& \widetilde{W}^{\mathrm{I}}\left(k_{1}, k_{2}, k_{3}, k_{4}\right)=4 \pi^{2} \int d^{4} p \theta\left(p^{0}\right) \delta\left(p^{2}-m^{2}\right) \times \\
& \times\left[\left\langle 0\left|\tilde{\mathrm{A}}\left(k_{1}\right) \widetilde{R}\left(-p / k_{2}\right)\right| 0\right\rangle+\left\langle 0\left|\widetilde{R}\left(-p / k_{1}\right) \tilde{\mathrm{A}}\left(k_{2}\right)\right| 0\right\rangle\right] \overleftarrow{\left(m^{2}-p^{2}\right)} \times \\
& \times \overleftrightarrow{\left(m^{2}-p^{2}\right)}\left[\left\langle 0\left|\tilde{\mathrm{A}}\left(k_{3}\right) \widetilde{R}\left(p / k_{4}\right)\right| 0\right\rangle+\left\langle 0\left|\widetilde{R}\left(p / k_{3}\right) \tilde{\mathrm{A}}\left(k_{4}\right)\right| 0\right\rangle\right] \\
& \text { für } 0<\left(k_{1}+k_{2}\right)^{2}<4 m^{2} .
\end{aligned}
$$

Es sei nun $\tilde{h}\left(p_{i}, p_{j}\right)$ eine Testfunktion aus $\mathfrak{\subseteq}_{8}$ mit der Trägereigenschaft:

$$
\tilde{h}\left(p_{i}, p_{j}\right)=0 \quad \text { falls } \quad\left(p_{i}+p_{j}\right)^{2} \geqq 4 m^{2} \quad \text { oder } \quad\left(p_{i}+p_{j}\right)^{2} \leqq 0 \quad \text { (95) }
$$

und $D_{m}$ diejenige Teilmenge aus $\mathfrak{G}$, die von Vektoren der Form

$$
\psi(h)=\int d^{4} x_{i} d^{4} x_{j} h\left(x_{i} ; x_{j}\right) \mathrm{A}\left(x_{i}\right) \mathrm{A}\left(x_{j}\right)|0\rangle
$$

aufgespannt wird, wobei die Funktionen $h(x, y)$ die Eigenschaft (95) haben. Dann folgt mit dem bekannten Reduktionsverfahren [20], [21], [37]:

$$
\begin{aligned}
& \int d^{4} x_{i} d^{4} x_{j} h\left(x_{i} ; x_{j}\right) \int d^{4} x \bar{f}_{\alpha}(x) K_{x}^{m}\left[\left\langle 0\left|R\left(x \mid x_{i}\right) \mathrm{A}\left(x_{j}\right)\right| 0\right\rangle+\right. \\
& \left.+\left\langle 0\left|\mathrm{~A}\left(x_{i}\right) R\left(x \mid x_{j}\right)\right| 0\right\rangle\right]=-i \int d^{4} x_{i} d^{4} x_{j} k\left(x_{i}, x_{j}\right) \lim _{t \rightarrow \infty} \int d_{x^{(0)}=t} x \overline{f_{\alpha}(x)} \frac{\overleftrightarrow{\partial}}{\partial x^{(0)}} \times \\
& \times\left\{\left\langle 0\left|\left[\mathrm{~A}(x) ; \mathrm{A}\left(x_{i}\right)\right] \mathrm{A}\left(x_{j}\right)\right| 0\right\rangle+\left\langle 0\left|\mathrm{~A}\left(x_{i}\right)\left[\mathrm{A}(x) ; \mathrm{A}\left(x_{j}\right)\right]\right| 0\right\rangle\right\} \\
& =-\int d^{4} x_{i} d^{4} x_{j} k\left(x_{i} ; x_{j}\right) \lim _{t \rightarrow+\infty}\left[\left\langle 0\left|\mathrm{~A}_{\alpha}^{f}(t) \mathrm{A}\left(x_{i}\right) \mathrm{A}\left(x_{j}\right)\right| 0\right\rangle\right. \\
& \left.-\left\langle 0\left|\mathrm{~A}\left(x_{i}\right) \mathrm{A}\left(x_{j}\right) \mathrm{A}_{\alpha}^{f}(t)\right| 0\right\rangle\right] .
\end{aligned}
$$

Infolge der schwachen asymptotischen Zustandskonvergenz auf $D_{m}\left(\mathfrak{S}^{\text {ex }} \leqq \mathfrak{G}[18] ;[19]\right.$ :

$$
w-\lim _{t \rightarrow+\infty} \mathrm{A}_{\alpha(t)^{*}}|0\rangle=\left(\mathrm{A}_{\text {out }}^{f_{\alpha}}\right)^{*}|0\rangle ; \mathrm{A}_{\text {out }}^{f_{\alpha}}|0\rangle=0
$$

erhalten wir daraus:

$$
\begin{aligned}
& \left(m^{2}-p^{2}\right)\left\{\left\langle 0\left|\widetilde{R}\left(p / k_{i}\right) \tilde{\mathrm{A}}\left(k_{j}\right)\right| 0\right\rangle+\left\langle 0\left|\tilde{\mathrm{A}}\left(k_{i}\right) \widetilde{R}\left(p / k_{j}\right)\right| 0\right\rangle\right\} \\
= & -\frac{1}{(2 \pi)}\left\{\begin{array}{llll}
\left\langle m ; p\left|\tilde{\mathrm{A}}\left(k_{i}\right) \tilde{\mathrm{A}}\left(k_{j}\right)\right| 0\right\rangle & \text { für } & p^{0}=+\sqrt{\vec{p}^{2}+m^{2}} \\
\left\langle 0\left|\tilde{\mathrm{A}}\left(k_{i}\right) \tilde{\mathrm{A}}\left(k_{j}\right)\right| p, m\right\rangle & \text { für } & p^{0}=-\sqrt{\vec{p}^{2}+m^{2}} .
\end{array}\right.
\end{aligned}
$$

Mit den drei letzten Gleichungen ergibt sich dann:

$$
\begin{aligned}
& \widetilde{W}^{\mathrm{I}}\left(k_{1}, k_{2}, k_{3}, k_{4}\right)= \int d^{4} p \theta\left(p^{0}\right) \delta\left(p^{2}-m^{2}\right) \times \\
& \times\left\langle 0\left|\tilde{\mathrm{A}}\left(k_{1}\right) \tilde{\mathrm{A}}\left(k_{2}\right)\right| p ; m\right\rangle\left\langle m ; p\left|\tilde{\mathrm{A}}\left(k_{3}\right) \tilde{\mathrm{A}}\left(k_{4}\right)\right| 0\right\rangle \\
& \text { für } 0<\left(k_{1}+k_{2}\right)^{2}<4 m^{2} .
\end{aligned}
$$

Dieser Ausdruck kompensiert aber gerade den Beitrag von $\widetilde{W}^{T}\left(k_{1}, \ldots, k_{4}\right)$ für $0<\left(k_{1}+k_{2}\right)^{2}<4 m^{2}$ in Gleichung (93). 
Durch die Forderungen (WI), (WIII) sowie (WII') für $W^{T}\left(x_{1}, \ldots, x_{4}\right)$ und $\left(\mathrm{WII}^{\prime \prime}\right)$ für $W^{\mathrm{II}}\left(x_{1}, \ldots, x_{4}\right)$ ist die in den Gleichungen $(91)-(93)$ angegebene Darstellung nicht eindeutig bestimmt. Wie nämlich die vorstehenden Überlegungen zeigen, können wir mittels der Ersetzung

$$
\begin{aligned}
\Delta_{\mathrm{ret}}(p) \rightarrow \Delta_{\mathrm{ret}}^{\prime}(p) & =\Delta_{\mathrm{ret}}(p)+\Re(p) \\
\Delta(p) \rightarrow \Delta^{\prime}(p) & =\Delta(p)+\Re(-p)-\Omega(p)
\end{aligned}
$$

in den Gleichungen $(91)-(94) \mathrm{zu}$ einer neuen Funktion $W_{\mathfrak{R}}^{I}\left(x_{1}, \ldots, x_{4}\right)$ gelangen, die ebenfalls die Bedingungen (WI), (WII') und (WIII) erfüllt, falls der Kern $\Re(p)$ die folgenden Eigenschaften hat:

$$
(\Omega)\left\{\begin{array}{l}
\Re(p)=\int_{4 m^{2}}^{\infty} \frac{d\left(\mu^{2}\right) \varrho\left(\mu^{2}\right)}{\mu^{2}-(p+i \varepsilon)^{2}} \\
\varrho\left(\mu^{2}\right)=0 \text { für } \mu^{2}<4 m^{2} .
\end{array}\right.
$$

Damit erhalten wir den Satz:

Satz IV. Für gegebene Funktionen $W\left(x_{1}, x_{1}, x_{3}\right)$ und $W^{T}\left(x_{1}, x_{2}, x_{3}, x_{4}\right)$ mit den Eigenschaften (WI), (WII') und (WIII) erfüllt die Funktion

$$
\widetilde{W}_{\mathfrak{R}}^{\mathrm{II}}\left(k_{1}, k_{2}, k_{3}, k_{4}\right)=W^{T}\left(k_{1}, k_{2}, k_{3}, k_{4}\right)-W_{\mathfrak{R}}^{\mathrm{I}}\left(k, k_{2}, k_{3}, k_{4}\right),
$$

wobei $W_{\Omega}^{\mathrm{I}}\left(k_{1}, k_{2}, k_{3}, k_{4}\right)$ durch die Gleichungen (91) und (99) definiert ist, die Bedingungen (WI), (WII") und (WIII) für jeden Kern $\Re(p)$ mit den Eigenschaften (囚). Die daraus konstruierte Funktion

$$
\bar{W}_{\Re}\left(x_{1}, x_{2}, x_{3}, x_{4}\right)=\prod_{r=1}^{4} K_{x r}^{m} W_{\mathfrak{R}}^{\mathrm{II}}\left(x_{1}, x_{2}, x_{3}, x_{4}\right)
$$

hat neben den Eigenschaften (WI) und (WIII) die Trägereigenschaft

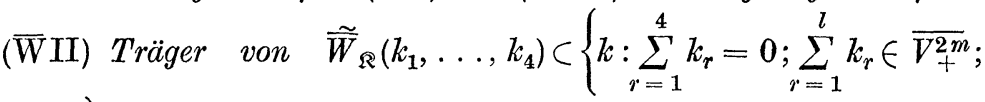
$l=1,2,3\}$.

Aus der Darstellung (91) folgt mit den Gleichungen (88), (92) und (93), daß die trunkierten Wightman-Funktionen in allen $k_{i}(i=1, \ldots, 4)$ und allen daraus gebildeten Partialsummen auf dem Massenhyperboloid eine Singularität $\delta\left(k^{2}-m^{2}\right)$ haben. Zu diesen kommen noch Hauptwertsingularitäten in den Variablen $k_{2}, k_{3}, k_{1}+k_{3}$ und $k_{2}+k_{3}$ hinzu.

Per Konstruktion tritt die Singularität $\delta\left(\left(k_{1}+k_{2}\right)^{2}-m^{2}\right)$ in $W_{\Omega}^{\mathrm{II}}\left(k_{1}, k_{2}, k_{3}, k_{4}\right)$ nicht mehr auf. Es besteht also die Frage, ob durch die obige Konstruktion weitere von den in den trunkierten WightmanFunktionen auftretenden Singularitäten automatisch aus $W_{\Omega}^{\mathrm{II}}$ eliminiert worden sind. Von den Singularitäten in $k_{2}$ und $k_{3}$ ist dies nicht zu erwarten, da diese Singularitäten auch in den $r^{\mathrm{I}}$-Funktionen noch enthalten sind. Wir werden am Ende von Abschnitt IV zeigen, daß $W_{\mathbb{R}}^{\text {II }}$ zumindest noch Hauptwertsingularitäten in $k_{2}$ und $k_{3}$ enthalten muß, 
da sonst das elastische Streumatrixelement ein Produkt von Dreipunktfunktionen wäre. Es liegt aber nun die Vermutung nahe, daß mit den Singularitäten in $k_{1}+k_{2}$ auch diejenigen in den restlichen Partialsummen $k_{1}+k_{3}$ bzw. $k_{2}+k_{3}$ in $W_{\mathfrak{R}}^{\mathrm{II}}$ nicht mehr auftreten, d. h., daß sie durch die Darstellung (91) und (100) auf die Integralkerne $\Delta_{\text {ret }}$ bzw. $\Delta_{+}$ zurückgeführt wären.

Um zu zeigen, da $\beta$ die $W_{\Omega}^{\mathrm{II}}$-Funktionen in keiner Partialsumme $\left(k_{\alpha}+k_{\beta}\right)$ $\alpha \neq \beta$ eine Singularität auf der Massenschale haben, müssen wir auf die $r$-Funktionen zurückgreifen. Wir werden zeigen, daß keine der zweiunddreißig $r^{\mathrm{I}}$-Funktionen in irgendeiner Partialsumme $\left(k_{\alpha}+k_{\beta}\right) 1 \leqq \alpha$; $\beta \leqq 4, \alpha \neq \beta$ auf der Massenschale eine Singularität hat. Da die Wightman-Funktionen in linearer Weise eindeutig durch die $r^{\mathrm{T}}$-Funktionen dargestellt werden können (falls letztere existieren), so folgt daraus, daß auch in den $W_{\mathfrak{\Omega}}^{\mathrm{II}}$-Funktionen diese Singularitäten nicht mehr auftreten.

Unter den Voraussetzungen (AI)-(AIV) und der L-S-Z-Asymptotenbedingung gelten nach SyмanzIK [22], [23] die folgenden Relationen für die gewöhnlichen $r$-Funktionen:

$$
\begin{gathered}
\tilde{r}\left(p / k_{1}, \ldots, k_{r}, q_{1}, \ldots q_{s}\right)=\left[m^{2}-\left(p+\sum_{j=1}^{r} k_{j}+i \varepsilon\right)^{2}\right]^{-1} \times \\
\times\left[m^{2}-\left(p+\sum_{j=1}^{r} k_{j}\right)^{2}\right] \tilde{r}\left(p / k_{1}, \ldots, k_{r}, q_{1}, \ldots, q_{s}\right) \\
\delta\left(m^{2}-\left(p+\sum_{j=1}^{r} k_{j}\right)^{2}\right)\left[m^{2}-\left(p+\sum_{j=1}^{r} k_{j}\right)^{2}\right]\left\{\tilde{r}\left(p / k_{1}, \ldots, k_{r}, q_{1}, \ldots, q_{s}\right)+\right. \\
\left.\quad+2 \pi \int d u \tilde{r}\left(p / k_{1}, \ldots, k_{r} ; u\right)\left(m^{2}-u^{2}\right) \tilde{r}\left(+u / q_{1}, \ldots, q_{s}\right)\right\}=0
\end{gathered}
$$

Daraus ergibt sich mit Gleichung (46) für die Vierpunktfunktion:

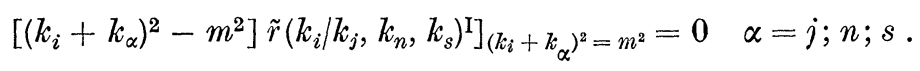

Aus diesen drei Gleichungen folgt aber mit der Symmetrie der $r$-Funktionen, daß $r_{i}(k)^{\mathrm{I}}$ und $a_{i}(k)^{\mathrm{I}}$ in keiner der Partialsummen $\left(k_{\alpha}+k_{\beta}\right)$ $\alpha \neq \beta$ eine Einteilchensingularität haben.

Mit Hilfe der in Abschnitt II hergeleiteten Regularitätseigenschaften der $r^{\mathrm{I}}$-Funktionen können wir zu den Gleichungen (102)-(104) analoge Relationen für die gemischten $r$-Funktionen beweisen, aus denen unmittelbar folgt, daß auch diese Funktionen keine Einteilchensingularitäten mehr enthalten.

Satz V. Unter den Voraussetzungen (AI)-(AIV) gilt:

$$
\begin{aligned}
\tilde{r}_{i j}(k)= & \frac{1}{m^{2}-\left(k_{\boldsymbol{i}}+k_{\beta}+i \varepsilon\right)^{2}}\left[m^{2}-\left(k_{i}+k_{\beta}\right)^{2}\right] \tilde{r}_{i j}(k) \quad \beta=j ; m ; n \\
& {\left.\left[m^{2}-\left(k_{i}+k_{\beta}\right)^{2}\right] \tilde{r}_{i j}(k)^{\mathrm{I}}\right]_{\left(k_{i}+k_{\beta}\right)^{2}=m^{2}}=0 \quad \beta=j ; m ; n . }
\end{aligned}
$$


Über die Einteilchensingularitäten in Relativistischen Quantenfeldtheorien 199

Beweis: Für $k \in B\left(m^{2} ; m^{2}\right)$ (reelle Regularitätspunkte von $G(p)$ ) gilt nach Satz II und Gleichung (102):

$$
\begin{gathered}
G(k)=\tilde{r}_{i}^{\prime}(k) \\
G(k)=\frac{1}{m^{2}-\left(k_{i}+k_{\beta}\right)^{2}}\left[m^{2}-\left(k_{i}+k_{\beta}\right)^{2}\right] G(k) \quad \beta=j ; m ; n .
\end{gathered}
$$

Durch analytische Fortsetzung beider Seiten dieser Gleichung und Limesbildung nach Satz II folgt die Gleichung (39) für beliebige $k$. Ebenso gilt nach Gleichung (103) für $k \in B\left(m^{2} ; 4 m^{2}\right)$ (reelle Regularitätspunkte von $\left.G(p)^{\mathrm{I}}\right)$ :

$$
\begin{gathered}
G(k)^{\mathrm{I}}=\tilde{r}_{i}^{\prime}(k)^{\mathrm{I}} \\
\left.\left[m^{2}-\left(k_{i}+k_{\beta}\right)^{2}\right] G(k)^{\mathrm{I}}\right]_{\left(k_{i}+k_{\beta}\right)^{2}=m^{2}=0}=j=j ; m ; n .
\end{gathered}
$$

Durch analytische Fortsetzung bei festgehaltenem $\left(k_{i}+k_{\beta}\right)^{2}=m^{2}$ in den restlichen unabhängigen Variablen und Limesbildung folgt dann Gleichung (106) für beliebige $k$.

Die Darstellungen (91) und (100) haben zunächst nur einen formalen Charakter, da die Matrixelemente der retardierten Kommutatoren mathematisch nicht präzise definiert sind. Im Anhang wird aber gezeigt, daß diese Matrixelemente mit Hilfe von verallgemeinerten Jost-LehmannDyson-Darstellungen bis auf ein Polynom im Impulsraum eindeutig definiert werden können [38] - [43]. In Gleichung (91) bzw. (100) verschwindet der Beitrag dieser Polynome auf der Massenschale infolge der auftretenden Klein-Gordon-Operatoren.

Am Ende von Abschnitt IV werden wir ohne Benutzung von retardierten Funktionen zeigen, daß $W_{\mathbb{R}}^{\mathrm{II}}$ keine Hauptwertsingularität in der Variablen $k_{2}+k_{3}$ enthält.

Das in den beiden letzten Abschnitten angegebene Konstruktionsverfahren läßt sich im Prinzip für beliebiges $n$ verallgemeinern. Leider ist es infolge kombinatorischer Schwierigkeiten bis jetzt nicht gelungen, eine zu (91) analoge Darstellung für $W_{\mathfrak{R}}^{\mathrm{I}}\left(x_{1}, \ldots, x_{n}\right)$ mit $n>4$ explizite anzugeben.

\section{Folgerungen aus der Asymptotenbedingung}

In diesem Abschnitt sollen unter der zusätzlichen Voraussetzung der LSZ-Asymptotenbedingung [19], [20], [21] die Aufspaltungen (7) und einige damit zusammenhängende Folgerungen bewiesen werden. Dazu stellen wir zunächst einmal diejenigen Asymptotenbedingungen zusammen, die nur aus den Annahmen (AI) - (A-IV) folgen.

Der Ausgangspunkt sind die Haagschen quasilokalen Operatoren [11]

$$
\mathrm{B}\left[h_{n}\right]=: \int d^{4} x_{1} \ldots d^{4} x_{n} h_{n}\left(x_{1} ; \ldots ; x_{n}\right) \mathrm{A}\left(x_{1}\right) \cdot \ldots \cdot \mathrm{A}\left(x_{n}\right),
$$


wobei $h_{n}$ ein Element aus $S_{4 n}$ ist.

$$
\begin{aligned}
\mathrm{B}\left[h_{n} / x\right] & =: \mathrm{T}(x) \mathrm{B}\left[h_{n}\right] \mathrm{T}(-x) \\
\tilde{\mathrm{B}}\left[h_{n} / p\right] & =(2 \pi)^{3 / 2} \int d^{4} p_{1} \ldots d^{4} p_{n} \times \\
& \times \delta\left(p-\sum_{j=1}^{n} p_{j}\right) \tilde{h}_{n}\left(p_{1} ; \ldots ; p_{n}\right) \prod_{j=1}^{n} \mathrm{~A}\left(p_{j}\right) .
\end{aligned}
$$

Ist nun der Träger von $s_{1}(p) \in \widetilde{S}_{4}$ um die Massenschale konzentriert, und ist $\left\{f_{\alpha}(x)\right\}$ ein vollständiges System von Lösungen positiver Frequenz der Klein-Gordon-Gleichung zur Masse $m$

$$
f_{\alpha}(x)=\frac{1}{(2 \pi)^{3 / 2}} \int d^{4} k \theta(k) \delta\left(k^{2}-m^{2}\right) \tilde{f}_{\alpha}(k) e^{-i k x},
$$

dann erzeugt der Operator

$$
\mathrm{B}_{g_{\alpha}}\left[s_{1} / t\right]^{*}=i \int_{t=x^{(0)}} d^{3} x \mathrm{~B}\left[s_{1} / x\right]^{*} \frac{\overleftrightarrow{\partial}}{\partial x^{(0)}} g_{\alpha}(x)
$$

mit

$$
\tilde{g}_{\alpha}(p)=\frac{\tilde{f}_{\alpha}(p)}{\left\langle m ; p\left|\mathrm{~B}\left[s_{1}\right]^{*}\right| 0\right\rangle}
$$

infolge der Spektrumsbedingung (AII) aus dem Vakuum einen Einteilchenzustand mit der Wellenfunktion $f_{\alpha}$ :

$$
\mathrm{B}_{g_{\alpha}}\left[s_{1} \mid t\right]^{*}|0\rangle=\left|\Phi_{f_{\alpha}}^{m}\right\rangle ; \mathrm{B}_{g_{\alpha}}\left[s_{1} \mid t\right]|0\rangle=0 .
$$

Ist allgemeiner $\mathfrak{S}_{4 n}^{m}$ der Raum der Testfunktionen $s_{n}(p) \in \mathfrak{S}_{4 n}$ mit der Eigenschaft:

$$
\text { Träger } s_{n}\left(p_{1}, \ldots, p_{n}\right) \leqq\left\{p: 0<\left(\sum_{j=1}^{n} p_{j}\right)^{2}<4 m^{2}\right\},
$$

dann gilt für alle Operatoren $\mathrm{B}\left[s_{n}\right]$, die den Bedingungen

$$
\begin{aligned}
& \left\langle m, p\left|\mathrm{~B}\left[s_{n}\right]^{*}\right| 0\right\rangle \neq 0 \\
& s_{n}\left(p_{1}, \ldots, p_{n}\right) \in \widetilde{\Xi}_{4 n}^{m}
\end{aligned}
$$

genügen, nach HAAG [11] und RUELLE [15] die starke asymptotische Zustandskonvergenz:

$$
\underset{t \rightarrow \pm \infty}{s-\lim _{j=1}} \prod_{j}^{m} \mathrm{~B}_{\alpha_{\alpha_{j}}}\left[S_{n_{j}} \mid t\right]^{*}|0\rangle=\prod_{j=1}^{m} \mathrm{~A}_{\text {in }}\left(f_{\alpha_{j}}\right)^{*}|0\rangle .
$$

Dabei ist $\mathrm{A}_{\mathrm{ex}}\left(f_{\alpha}\right)$ mit $\mathrm{ex}=$ in oder out ein freies Feld zur Masse $m$

$$
\begin{aligned}
\mathrm{A}_{\mathrm{ex}}\left(f_{\alpha}\right) & =i \int d^{3} x \overline{f_{\alpha}(x)} \frac{\overleftrightarrow{\partial}}{\partial x^{(0)}} \mathrm{A}_{\mathrm{ex}}(x) \\
\mathrm{A}_{\mathrm{ex}}(x) & =\frac{1}{(2 \pi)^{3 / 2}} \int d^{4} k \delta\left(k^{2}-m^{2}\right) \tilde{\mathrm{A}}_{\mathrm{ex}}(k) l^{-i k x} \\
\mathrm{~A}_{\mathrm{ex}}(k) & =\theta(k) a_{\mathrm{ex}}(\mathbf{k})+\theta(-k) a_{\mathrm{ex}}(-\mathbf{k})^{*} \\
{\left[a_{\mathrm{ex}}(\mathbf{k}) ; a_{\mathrm{ex}}\left(\mathbf{k}^{\prime}\right)^{*}\right] } & =2 \omega_{k} \delta\left(\mathbf{k}-\mathbf{k}^{\prime}\right) ; \omega_{k}=+\sqrt{\mathbf{k}^{2}+m^{2}}
\end{aligned}
$$


Die lineare abgeschlossene Hülle der Vektoren $\prod_{r=1}^{l} \mathrm{~A}_{\mathrm{ex}}\left(f_{\alpha_{r}}\right) *|0\rangle$ ergibt die asymptotischen Hilberträume $\mathfrak{G}^{\mathrm{ex}} \leqq \mathfrak{Y}$.

Für beliebiges $h_{n}(p) \in \mathfrak{\Im}_{4 n}$ konnten HAAG und RoBINson [44] und unabhängig davon HEPP [19] eine schwache asymptotische Operatorkonvergenz auf einer in $\mathfrak{F}^{\text {ex }}$ dichten Teilmenge beweisen. Darüber hinaus hat HEPP [19] die folgende Form der Yang-Feldman-Gleichung hergeleitet:

Die Funktionen $\hat{\varphi}_{n}\left(\mathbf{k}_{1}, \ldots, \mathbf{k}_{n}\right)$ seien definiert durch

$$
\hat{\varphi}_{n}\left(\mathbf{k}_{1}, \ldots, \mathbf{k}_{n}\right)=\frac{1}{\sqrt{n !}} \sum_{\text {Perm. }\{1, \ldots, n\}} \tilde{\varphi}_{n}\left(\omega_{k_{1}}, \mathbf{k}_{1}, \ldots, \omega_{k_{n}}, \mathbf{k}_{n}\right),
$$

wobei $\tilde{\varphi}_{n}\left(k_{1}, \ldots, k_{n}\right)$ eine Funktion aus $\mathfrak{\Im}_{4 n}$ der Art ist, daß für alle $k_{j}$ aus ihrem Träger paarweise die Bedingung

$$
\mathbf{k}_{i} \omega_{k i}^{-1} \neq \mathbf{k}_{j} \omega_{k j}^{-1} \text { für } i \neq j
$$

erfüllt ist. Weiterhin sei $D^{\text {ex }}$ die in $\mathfrak{F}^{\text {ex }}$ dichte Menge, die von beliebigen Linearkombinationen von Vektoren der Art

$$
\Phi_{\mathrm{ex}}\left(\hat{\varphi}_{n}\right)=\int \frac{d^{3} p_{1}}{2 \omega p_{1}} \cdots \frac{d^{3} p_{n}}{2 \omega p_{n}} \hat{\varphi}_{n}\left(\mathbf{p}_{1}, \ldots, \mathbf{p}_{n}\right) \prod_{j=1}^{n} a_{\mathrm{ex}}\left(\mathbf{p}_{j}\right)^{*}|0\rangle
$$

aufgespannt wird. Dann gilt für $\Phi_{\text {ex }} \in D^{\text {ex }}$ die Yang-Feldman-Gleichung

$$
\begin{aligned}
& \tilde{\mathrm{B}}\left[h_{n} / p\right] \underset{\text { in }}{\Phi_{\text {out }}}=\delta\left(p^{2}-m^{2}\right) \\
& \underset{\text { in }}{\tilde{\mathrm{B}}_{\text {int }}}\left[h_{n} / p\right] \underset{\text { in }}{\text { out }} \\
& \times\left[p^{2}-m^{2}\right] \tilde{\mathrm{B}}\left[h_{n} / p\right] \Phi_{\text {int }}^{\text {out }}
\end{aligned}
$$

$\tilde{\mathrm{B}}_{\mathrm{ex}}\left[h_{n} / p\right]=\theta(p)\left\langle 0\left|\mathrm{~B}\left[h_{n}\right]\right| p, m\right\rangle a_{\mathrm{ex}}(\mathbf{p})+\theta(-p)\langle m,-p| \times$

$\times \mathrm{B}\left[h_{n}\right]|0\rangle a_{\mathrm{ex}}(-\mathbf{p})^{*}$

als Identität in $\varsigma_{4}^{\prime}[19]$.

Werden die quasilokalen Operatoren $\mathrm{B}\left[h_{n}\right]$ nicht aus Produkten von lokalen Feldern $\mathrm{A}(x)$ gebildet, sondern sind sie regularisierte Elemente von lokalen Observablenringen, dann können in der Gleichung (121) die Zustände $\Phi_{\text {ex }}(\hat{\varphi})$ durch beliebige Zustände $\Psi \in \mathfrak{G}$ zu beschränkter Energie ersetzt werden [18].

Für die weiteren U̇berlegungen müssen wir nun zwei zusätzliche Annahmen machen:

(AV) Asymptotische Vollständigkeit: $\mathfrak{G}^{\mathrm{ex}}=\mathfrak{S}$.

(AVI) Auf allen Zuständen der Art

$$
\Psi\left(f_{m}\right)=\int d^{4} q_{1} \ldots d^{4} q_{m} f_{m}\left(q_{1}, \ldots, q_{m}\right) \prod_{j=1}^{m} \tilde{\mathrm{A}}\left(q_{j}\right)|0\rangle
$$


mit $f_{m} \in \widetilde{S}_{4 m}$ gelten die Yang-Feldman-Gleichungen

$$
\begin{aligned}
& \theta(p) \delta\left(p^{2}-m^{2}\right)\left\{a_{\text {out }}(\mathbf{p})-a_{\text {in }}(\mathbf{p})\right\} \Psi= \\
& \quad-\frac{2 \pi i \theta(p) \delta\left(p^{2}-m^{2}\right)}{\left\langle 0\left|\mathrm{~B}\left[h_{n}\right]\right| p_{i} m\right\rangle}\left[p^{2}-m^{2}\right] \tilde{\mathrm{B}}\left[h_{n} / p\right] \Psi \\
& \theta(-p) \delta\left(p^{2}-m^{2}\right)\left\{a_{\text {out }}(\mathbf{p})^{*}-a_{\text {in }}(\mathbf{p})^{*}\right\} \Psi= \\
& \quad+\frac{2 \pi i \theta(-p) \delta\left(p^{2}-m^{2}\right)}{\left\langle m_{i}-p\left|\mathrm{~B}\left[h_{n}\right]\right| 0\right\rangle}\left[p^{2}-m^{2}\right] \tilde{\mathrm{B}}\left[h_{n} / p\right] \Psi
\end{aligned}
$$

als Identitäten in $\mathfrak{\Xi}_{4}^{\prime}$.

Anmerkung $^{2}$ : Die Voraussetzung $f_{m}$ beliebig aus $\mathfrak{S}_{4 m}$ wird nur für die Aussagen über die Einteilchensingularitäten der Wightman-Funktionen gebraucht. Für alle daraus hergeleiteten Folgerungen über die S-Matrix oder deren Matrixelemente genügt die schwächere Annahme, daß in den Zuständen $\Psi\left(f_{m}\right)$ nur solche Funktionen $f_{m} \in \widetilde{S}_{4 m}$ auftreten, die für alle $k_{j}$ aus ihrem Träger paarweise die Bedingung

erfüllen.

$$
\mathbf{k}_{i}\left|k_{j}^{(0)}\right| \neq \mathbf{k}_{j}\left|k_{i}^{(0)}\right| \quad \text { für } \quad i \neq j
$$

Außerdem soll das Feld folgendermaßen normiert werden:

$$
\langle 0|\mathrm{~A}(0)| p, m\rangle=\frac{1}{2 \pi^{3 / 2}} \rightarrow\left\langle 0\left|\mathrm{~B}\left[h_{1}\right]\right| p, m\right\rangle=\tilde{h}_{1}(p),
$$

so daß gilt:

$$
\begin{aligned}
& \delta\left(p^{2}-m^{2}\right)\left\{\tilde{\mathrm{A}}_{\text {out }}(p)-\tilde{\mathrm{A}}_{\text {in }}(p)\right\} \Psi= \\
& \quad-(2 \pi)^{5 / 2} i \varepsilon(p) \delta\left(p^{2}-m^{2}\right)\left[p^{2}-m^{2}\right] \tilde{\mathrm{A}}(p) \Psi .
\end{aligned}
$$

Bilden wir nun das Matrixelement von (123) mit zwei Zuständen der Art (122), so erhalten wir mit

$$
|p, m\rangle=a_{\mathrm{ex}}(\mathbf{p})^{*}|0\rangle=\tilde{\mathrm{A}}_{\mathrm{ex}}(-p)|0\rangle \quad p^{(0)}>0
$$

und unter Berücksichtigung des Schwartzschen Kernsatzes [1] die folgende Identität auf $\mathfrak{S}_{4(n+m+r+1)}$ :

$$
\begin{aligned}
\delta\left(p^{2}\right. & \left.-m^{2}\right)\left\{(2 \pi)^{5 / 2} i \varepsilon(p) \delta\left(p-\sum_{j=1}^{n} p_{j}\right)\left(p^{2}-m^{2}\right) W_{r+n+l} \times\right. \\
& \times\left(k_{1}, \ldots, k_{r}, p_{1}, \ldots, p_{n}, q_{1}, \ldots, q_{l}\right)- \\
& -\left[\left\langle 0\left|\tilde{\mathrm{A}}\left(p_{1}\right) \ldots \mathrm{A}\left(p_{n}\right) \tilde{\mathrm{A}}_{\mathrm{ex}}(-p)\right| 0\right\rangle+\right. \\
& \left.+\left\langle 0\left|\tilde{\mathrm{A}}_{\mathrm{ex}}(-p) \tilde{\mathrm{A}}\left(p_{1}\right) \ldots \tilde{\mathrm{A}}\left(p_{n}\right)\right| 0\right\rangle\right] \times \\
& \left.\times\left\langle 0\left|\tilde{\mathrm{A}}\left(k_{1}\right) \ldots \tilde{\mathrm{A}}(k)\left[\tilde{\mathrm{A}}_{\text {out }}(p)-\tilde{\mathrm{A}}_{\text {in }}(p)\right] \tilde{\mathrm{A}}\left(q_{1}\right) \ldots \tilde{\mathrm{A}}\left(q_{l}\right)\right| 0\right\rangle\right\}=0
\end{aligned}
$$
Punkt.

${ }^{2}$ Ich danke Herrn Prof. R. HAAG für eine wertvolle Diskussion zu diesem 
Über die Einteilchensingularitäten in Relativistischen Quantenfeldtheorien 203 oder mit Gleichung (126):

$$
\begin{gathered}
\varepsilon(p) \delta\left(p^{2}-m^{2}\right)\left\{\delta\left(p-\sum_{j=1}^{n} p_{j}\right)\left[p^{2}-m^{2}\right] \widetilde{W}_{r+n+l}\right. \\
\left(k_{1}, \ldots, k_{r}, p_{1}, \ldots p_{n}, q_{1} ; \ldots q_{s}\right)- \\
-\left[\left\langle 0\left|\tilde{\mathrm{A}}\left(p_{1}\right) \ldots \mathrm{A}\left(p_{n}\right) \tilde{\mathrm{A}}_{\mathrm{ex}}(-p)\right| 0\right\rangle+\left\langle 0\left|\tilde{\mathrm{A}}_{\mathrm{ex}}(-p) \tilde{\mathrm{A}}\left(p_{1}\right) \ldots \tilde{\mathrm{A}}\left(p_{n}\right)\right| 0\right\rangle\right] \times \\
\left.\times\left(p^{2}-m^{2}\right) \widetilde{W}_{r+l+1}\left(k_{1}, \ldots, k_{r}, p, q_{1}, \ldots q_{l}\right)\right\}=0 .
\end{gathered}
$$

Aus der letzten Gleichung erhalten wir mit

$$
\delta\left(p^{2}-m^{2}\right)\left\langle 0\left|\tilde{\mathrm{A}}_{\mathrm{ex}}(p)\right| p ; m\right\rangle=\langle 0|\tilde{\mathrm{A}}(p)| p, m\rangle
$$

sehr leicht die Gleichung (7).

Weiterhin folgt aus Gleichung (128), daß

$$
\begin{aligned}
& F(p)=\int \prod_{\alpha=1}^{r} d^{4} k_{\alpha} \prod_{\beta=1}^{n} d^{4} p_{\beta} \prod_{\delta=1}^{l} d q_{\delta} \tilde{f}_{r+n+l}\left(k_{1}, \ldots, k_{r}, p_{1}, \ldots p_{n}, q_{1}, \ldots q_{l}\right) \times \\
& \times \delta\left(p-\sum_{j=1}^{n} p_{j}\right)\left[m^{2}-\left(\sum_{j=1}^{n} p_{j}\right)^{2}\right] \widetilde{W}_{r+n+l}\left(k_{1}, \ldots, k_{r}, p_{1}, \ldots p_{n}, q_{1}, \ldots q_{l}\right)
\end{aligned}
$$

für $f_{l+n+r} \in \mathfrak{S}_{4 l} \times \mathfrak{S}_{4 n}^{m} \times \mathfrak{S}_{4 r}$ eine stetige eigentliche Funktion ist. Mit anderen Worten $\widetilde{W}\left(k_{1}, \ldots, k_{r}, p_{1}, \ldots, p_{n}, q_{1}, \ldots, q_{s}\right)$ kann auf der Massenschale höchstens wie $\left[\left(\sum_{j=1}^{n} p_{j}\right)^{2}-m^{2}\right]^{-1}$ singulär werden.

Definieren wir die S-Matrix durch $A_{\text {out }}=S^{-1} A_{\text {in }} S$, so ergibt sich ebenfalls aus Gleichung (128) für $n=1$ :

Satz VI. Die S-Matrix ist dann und nur dann vom Einheitsoperator verschieden, wenn irgendein $\widetilde{W}_{r}\left(p_{1}, \ldots, p_{r}\right)$ mit $r \geqq 3$ in mindestens einem der Impulse $p_{j}$ mit $1<j<r$ eine Singularität der Art $\left(p_{j}^{2}-m^{2}\right)^{-1}$ hat.

Aus Gleichung (129) folgt dann schließlich:

Satz VII. Hat $\widetilde{W}_{r+n+1}\left(k_{1}, \ldots, k_{r}, p_{1}, \ldots, p_{n}, q_{1}, \ldots, q_{l}\right)$ für festes $r, l$ und irgendein beliebiges $n$ auf der Massenschale eine Singularität der $\operatorname{Art}\left[\left(\sum_{j=1}^{n} p_{j}\right)^{2}-m^{2}\right]^{-1}$, dann haben alle $\widetilde{W}_{r+j+1}\left(k_{1}, \ldots, k_{r}, p_{1}, \ldots, p_{j}\right.$, $\left.q_{1}, \ldots, q_{l}\right)$ für alle $j$, für die $\left\langle 0\left|\prod_{i=1}^{n} \mathrm{~A}\left(p_{i}\right)\right| 1\right\rangle \neq 0$ ist, ebenfalls eine Singularität der Art $\left[\left(\sum_{i=1}^{j} p_{i}\right)^{2}-m^{2}\right]^{-1}$.

Als eine Folgerung aus den beiden letzten Sätzen erhalten wir schließlich den Satz von Greenberg und Licht [45], [46]. 
Satz VIII. (Greenberg und LICHT): Verschwinden die trunkierten Wightman-Funktionen $W_{n}^{T}\left(k_{1}, \ldots, k_{n}\right)$ für alle $n>N$, so ist die S-Matrix ein Vielfaches der Einheitsmatrix.

Beweis: Aus der Voraussetzung $W_{n}^{T}\left(k_{1}, \ldots, k_{n}\right)=0$ für alle $n>N$ folgt zunächst

$$
\begin{aligned}
& \delta\left(p^{2}-m^{2}\right) \varepsilon\left(p^{0}\right) \delta\left(p-\sum_{j=1}^{n} p_{j}\right)\left(p^{2}-m^{2}\right) \\
& \widetilde{W}_{r+n+l}\left(k_{1}, \ldots, k_{r}, p_{1}, \ldots p_{n} ; q_{1} ; \ldots, q_{l}\right)=0
\end{aligned}
$$

für alle $n>N$ und beliebiges $r, l$.

Denn diese Wightman-Funktionen sind Produkte von trunkierten Wightman-Funktionen $W_{s}^{T}\left(k_{1}, \ldots, k_{s}\right)$ mit $s<N$, die keine Singularität der Art $\left[\left(\sum_{j=1}^{n>N} k_{j}\right)^{2}-m^{2}\right]^{-1}$ haben können.

Da andererseits mit $\left\langle 0\left|\mathrm{~A}\left(p_{1}\right)\right| k, m\right\rangle \neq 0$ auch gilt:

$$
\left\langle 0\left|\tilde{\mathrm{A}}\left(p_{1}\right) \ldots \tilde{\mathrm{A}}\left(p_{n}\right)\right| k, m\right\rangle \neq 0 \text { für } n>2,
$$

so ergibt sich aus Gleichung (128) für $n>N$ :

$$
\begin{aligned}
& \quad \varepsilon(p) \delta\left(p^{2}-m^{2}\right)\langle 0| \tilde{\mathrm{A}}\left(k_{1}\right) \ldots \tilde{\mathrm{A}}\left(k_{r}\right) \times \\
& \times\left[\tilde{\mathrm{A}}_{\text {out }}(p)-\tilde{\mathrm{A}}_{\text {in }}(p)\right] \tilde{\mathrm{A}}\left(q_{1}\right) \ldots \tilde{\mathrm{A}}\left(q_{e}\right)|0\rangle=0 .
\end{aligned}
$$

Da diese Gleichung für eine in $\mathfrak{S}$ dichte Menge gilt, so folgt mit der Unitarität von S für alle $\Psi_{\text {out }}, \Phi_{\text {out }} \in\left\{\right.$ Polynom $\left.\left[\mathrm{A}_{\text {out }}\right]|0\rangle\right\}$ :

$$
\left\langle\Psi_{\text {out }} \mid \mathrm{SA}_{\text {out }} \Phi_{\text {out }}\right\rangle-\left\langle\mathrm{A}_{\text {out }}^{*} \Psi_{\text {out }} \mid \mathrm{S} \Phi_{\text {out }}\right\rangle=0 \text {. }
$$

Wie die vorstehenden Überlegungen zeigen, ist für die Nichttrivialität einer unitären S-Matrix notwendig, daß in den Wightman-Funktionen Singularitäten der Art $\left[\left(\sum_{r=1}^{n} k_{r}\right)^{2}-m^{2}\right]^{-1}$ für beliebig großes $n$ auftreten. Daraus folgt aber, daß die in Abschnitt I erwähnten Modelle, die man durch die Definition $W_{\Omega}^{\text {II }}\left(k_{1}, \ldots, k_{r}\right)=0$ für $r>N$ erhält, entweder eine nicht unitäre oder eine triviale S-Matrix haben. Denn in diesem Fall sind alle Wightman-Funktionen „Produkte“ von $W_{\mathbb{R}}^{\mathrm{II}}\left(k_{1}, \ldots, k_{s}\right)$ Funktionen mit $s \leqq N$. Dann können sie aber keine Singularitäten der obigen Art mit $n>N$ haben.

Setzen wir voraus, daß nicht alle trunkierten Wightman-Funktionen ab einer gewissen Ordnung, sondern daß nur ein einziges $\widetilde{W}_{N}^{T}\left(k_{1}, \ldots, k_{N}\right)$ mit $N \geqq 4$ identisch verschwindet, so verschwinden die Streuamplituden für Übergänge aus einem Anfangszustand, bestehend aus zwei Teilchen, in einen Endzustand, bestehend aus $n$ Teilchen für alle $n$. Diese Aussage ist eine unmittelbare Folgerung aus dem folgenden Satz: 
Über die Einteilchensingularitäten in Relativistischen Quantenfeldtheorien 205

Satz IX. Falls $\widetilde{W}_{N}^{T}\left(k_{1}, \ldots, k_{N}\right)=0$ für irgendein $N>3$ und falls $\left\langle 0\left|\tilde{\mathrm{A}}\left(k_{1}\right) \tilde{\mathrm{A}}\left(k_{2}\right)\right| p, m\right\rangle \neq 0$, dann gilt:

$$
\begin{aligned}
& \delta\left(\left(\sum_{j=1}^{n} p_{j}\right)^{2}-m^{2}\right)\left[\left(\sum_{j=1}^{n} p_{j}\right)^{2}-m^{2}\right] \times \\
& \times\left\langle 0\left|\prod_{\alpha=0}^{r} \tilde{\mathrm{A}}\left(k_{\alpha}\right) \prod_{j=1}^{n} \tilde{\mathrm{A}}\left(p_{j}\right) \prod_{\beta}^{S} \tilde{\mathrm{A}}\left(q_{\beta}\right)\right| 0\right\rangle=0
\end{aligned}
$$

für beliebiges $n$ und alle $r, s$ mit $0 \leqq r+s \leqq N$.

Beweis: Zunächst folgt aus der Voraussetzung durch Entwicklung nach trunkierten Wightman-Funktionen:

$$
\delta\left(\left(\sum_{j=1}^{N-2} p_{j}\right)^{2}-m^{2}\right)\left[\left(\sum_{j=1}^{N-2} p_{j}\right)^{2}-m^{2}\right]\left\langle 0\left|\tilde{\mathrm{A}}\left(k_{1}\right) \prod_{j=1}^{N-2} \tilde{\mathrm{A}}\left(p_{j}\right) \tilde{\mathrm{A}}\left(q_{1}\right)\right| 0\right\rangle=0 .
$$

Daraus ergibt sich mit Satz VII:

$$
\delta\left(\left(\sum_{j=1}^{n} p_{j}\right)^{2}-m^{2}\right)\left[\left(\sum_{j=1}^{n} p_{j}\right)^{2}-m^{2}\right]\left\langle 0\left|\tilde{\mathrm{A}}\left(k_{1}\right) \prod_{j=1}^{n} \tilde{\mathrm{A}}\left(p_{j}\right) \tilde{\mathrm{A}}\left(q_{1}\right)\right| 0\right\rangle=0
$$

für beliebiges $n$.

Diese Gleichung gilt aber auch für die entsprechenden trunkierten Wightman-Funktionen. Nach Voraussetzung folgt aus den beiden letzten Gleichungen wieder durch Entwicklung nach trunkierten Wightman-Funktionen:

$\delta\left(\left(\sum_{j=1}^{n} p_{j}\right)^{2}-m^{2}\right)\left[\left(\sum_{j=1}^{n} p_{j}\right)^{2}-m^{2}\right]\left\{\begin{array}{c}\left\langle 0\left|\tilde{\mathrm{A}}\left(k_{1}\right) \tilde{\mathrm{A}}\left(k_{2}\right) \prod_{j=1}^{n} \tilde{\mathrm{A}}\left(p_{j}\right) \tilde{\mathrm{A}}\left(q_{1}\right)\right| 0\right\rangle \\ \left\langle 0\left|\tilde{\mathrm{A}}\left(k_{1}\right) \prod_{j=1}^{n} \tilde{\mathrm{A}}\left(p_{j}\right) \tilde{\mathrm{A}}\left(q_{1}\right) \tilde{\mathrm{A}}\left(q_{2}\right)\right| 0\right\rangle\end{array}\right\}=0$ zunächst für $n=N-3$, und daraus dann mit Satz VII für beliebiges $n$. Durch Weiterführung dieses Verfahrens ergibt sich sukzessive die Behauptung.

Satz X. Falls $\left\langle 0\left|\tilde{\mathbf{A}}\left(k_{1}\right) \tilde{\mathrm{A}}\left(k_{2}\right)\right| p, m\right\rangle \neq 0$ und falls $\widetilde{W}_{N}^{T}\left(k_{1}, \ldots, k_{N}\right)=0$ oder $\delta\left(\left(\sum_{j=2}^{N-2} k_{j}\right)^{2}-m^{2}\right)\left[\left(\sum_{j=2}^{N-2} k_{j}\right)^{2}-m^{2}\right] \widetilde{W}_{N}\left(k_{1}, \ldots, k_{N}\right)=0$ für irgendein $N>3$, so gilt für die Übergangsmatrixelemente $T_{2 \rightarrow n}$ für beliebiges $n$ :

$$
\begin{aligned}
T_{2 \rightarrow n n}\left(\bar{g}_{1}, \ldots, \bar{g}_{n} \mid\right. & \left.g_{n+1}, g_{n+2}\right)=: \mathrm{S}_{2 \rightarrow n}\left(\bar{g}_{1}, \ldots, \bar{g}_{n} \mid g_{n+1}, g_{n+2}\right)- \\
& -\left\langle 0\left|\prod_{j=1}^{n} \mathrm{~A}_{\text {out }}\left(g_{j}\right) \mathrm{A}_{\text {out }}\left(g_{n+1}\right)^{*} \mathrm{~A}_{\text {out }}\left(g_{n}\right)^{*}\right| 0\right\rangle=0 .
\end{aligned}
$$

Beweis: Für das Ưbergangsmatrixelement aus einem Anfangszustand, bestehend aus zwei Teilchen mit den Wellenfunktionen $g_{n+1}(k)$ und $g_{n+2}(k)$, in einen Endzustand, bestehend aus $n$ Teilchen mit den Wellenfunktionen $g_{1}(k), \ldots, g_{n}(k)$ ergibt sich durch Anwendung der 
Yang-Feldman-Gleichung (121) und der Schwartzschen Ungleichung die folgende Majorisierung:

$$
\begin{aligned}
&\left|T_{2 \rightarrow n}\right| \leqq(2 \pi)^{5 / 2} \| \prod_{j=1}^{n} \mathrm{~A}_{\text {out }}\left(g_{j}\right)^{*}|0\rangle \| \cdot\left[\int d^{4} q_{1} d^{4} q_{2} \overline{g_{n+1}\left(q_{1}\right)} g_{n+1}\left(q_{2}\right) \times\right. \\
& \times \prod_{r=1}^{2} \theta\left(q_{r}\right) \delta\left(q_{r}^{2}-m^{2}\right)\left(q_{r}^{2}-m^{2}\right) \times \\
&\left.\times\left\langle 0\left|\mathrm{~A}_{\text {ex }}\left(g_{n+2}\right) \tilde{\mathrm{A}}\left(q_{1}\right) \tilde{\mathrm{A}}\left(-q_{2}\right) \mathrm{A}_{\text {ex }}\left(g_{n+2}\right)^{*}\right| 0\right\rangle\right]^{1 / 2}
\end{aligned}
$$

Aus dieser Ungleichung folgt mit Satz IX bzw. Satz VII sofort die Behauptung.

Für den Beweis der vorstehenden Sätze VI-X sind weder die Lokalität noch die Lorentzkovarianz der Felder notwendige Voraussetzungen. Diese Sätze gelten für alle Feldtheorien, für die die Yang-FeldmanGleichungen gelten, wie z. B. in den nicht kovarianten, quasilokalen Theorien von HAAG [31] und ARAKI [18].

Es sollen nun noch die am Ende von Abschnitt III aufgestellten Behauptungen über die Singularitäten von $\widetilde{W}_{\mathbb{\Omega}}^{\mathrm{II}}\left(k_{1}, \ldots, k_{4}\right)$ bewiesen werden.

Für die elastische Streuamplitude $T_{2 \rightarrow 2}$ ergibt sich mit Hilfe der Yang-Feldman-Gleichung (121) die folgende Darstellung [47]:

$$
\begin{aligned}
T_{2 \rightarrow 2}\left(\bar{g}_{1}, \bar{g}_{2} \mid g_{3}, g_{4}\right) \\
=-i(2 \pi)^{5 / 2} \lim _{t \rightarrow+\infty} \int \prod_{j=1}^{4} d^{4} k_{j} \overline{g_{1}\left(k_{1}\right)} \overline{g_{2}\left(k_{2}\right)} g_{3}\left(k_{3}\right) g_{4}\left(k_{4}\right) \times \\
\quad \times \delta\left(k_{1}^{2}-m^{2}\right) \delta\left(k_{4}^{2}-m^{2}\right) \theta\left(k_{3}\right) \delta\left(k_{3}^{2}-m^{2}\right)\left(k_{3}^{2}-m^{2}\right) \times \\
\quad \times\left\langle 0\left|\tilde{\mathrm{A}}_{\mathrm{ex}}\left(k_{1}\right) \tilde{\mathrm{A}}\left(k_{2}\right) \tilde{\mathrm{A}}\left(-k_{3}\right) \tilde{\mathrm{A}}_{\mathrm{ex}}\left(-k_{4}\right)\right| 0\right\rangle \frac{k^{(0)}-\omega k_{2}}{2 \omega k_{2}} e^{-i\left(k_{2}^{0}-\omega_{k_{2}}\right) t} .
\end{aligned}
$$

Setzen wir die Gleichungen (91) und (100) für den Vakuumerwartungswert ein, so folgt wegen des auftretenden Klein-Gordon-Operators $\left(k_{3}^{2}-m^{2}\right)$, daß das Matrixelement $T_{2 \rightarrow 2}$ ein Produkt von Dreipunktfunktionen ist, falls $W_{\mathbb{R}}^{\mathrm{II}}\left(k_{1}, k_{2}, k_{3}, k_{4}\right)$ keine Hauptwertsingularität in $k_{3}$ und damit auch in $k_{2}$ hat.

Die Aussage über die Hauptwertsingularität in $k_{2}+k_{3}$ ergibt sich aus dem folgenden Satz:

Satz XI. Unter den Voraussetzungen (A. I) - (A. VI) gilt für die durch Gleichung $(100)$ definierte Funktion $W_{\Omega}^{\mathrm{II}}\left(p_{1}, p_{2}, p_{3}, p_{4}\right)$ :

$\delta\left(\left(p_{2}+p_{3}\right)^{2}-m^{2}\right) \varepsilon\left(p_{2}+p_{3}\right)\left[\left(p_{2}+p_{3}\right)^{2}-m^{2}\right] \widetilde{W}_{\Omega}^{\mathrm{II}}\left(p_{1}, p_{2}, p_{3}, p_{4}\right)=0$

Beweis: Zunächst folgt aus den Gleichungen (86), (91) und (100) mit den Trägereigenschaften der auftretenden Vakuumerwartungswerte sowie mit

$$
\left[\left(p_{2}+p_{3}\right)^{2}-m^{2}\right]\left\{\begin{array}{l}
\widetilde{W}\left(p_{2}, p_{3}, k\right) \\
\widetilde{W}\left(k, p_{2}, p_{3}\right)
\end{array}=0 \text { für } \quad 0<\left(p_{2}+p_{3}\right)^{2}<4 m^{2}\right.
$$


die folgende Relation:

$$
\begin{aligned}
\varepsilon(p) & \delta\left(p^{2}-m^{2}\right) \delta\left(p-p_{2}-p_{3}\right)\left(p^{2}-m^{2}\right)\left\{\widetilde{W}^{T}\left(p_{1}, p_{2}, p_{3}, p_{4}\right)-\right. \\
& -\widetilde{W}_{\mathbb{R}}^{\mathrm{II}}\left(p_{1}, p_{2}, p_{3}, p_{4}\right)+2 \pi \int d^{4} k \widetilde{W}\left(p_{1},-k, p_{4}\right) \overleftarrow{\left(m^{2}-k^{2}\right)} \times \\
& \left.\times\left[\left\langle 0\left|\tilde{\mathrm{A}}\left(p_{2}\right) \widetilde{R}\left(k / p_{3}\right)\right| 0\right\rangle+\left\langle 0\left|\widetilde{R}\left(k / p_{2}\right) \tilde{\mathrm{A}}\left(p_{3}\right)\right| 0\right\rangle\right]\right\}=0 .
\end{aligned}
$$

Mit $\left(p_{2}+p_{3}\right)^{2}=m^{2}$ gilt auch $k^{2}=m^{2}$. Mit Gleichung (97) folgt dann:

$$
\begin{aligned}
\delta\left(p^{2}-m^{2}\right)\left\{\delta ( p - p _ { 2 } - p _ { 3 } ) \varepsilon ( p ) ( p ^ { 2 } - m ^ { 2 } ) \left[\tilde{W}^{T}\left(p_{1}, p_{2}, p_{3}, p_{4}\right)-\right.\right. \\
\left.\quad-\widetilde{W}_{\mathbb{R}}^{\mathrm{II}}\left(p_{1}, p_{2}, p_{3}, p_{4}\right)\right]-\tilde{W}\left(p_{1}, p, p_{4}\right) \overleftarrow{\left(p^{2}-m^{2}\right)} \times \\
\left.\quad \times\left[\left\langle 0\left|\tilde{\mathrm{A}}\left(p_{2}\right) \tilde{\mathrm{A}}\left(p_{3}\right) \tilde{\mathrm{A}}_{\mathrm{ex}}(-p)\right| 0\right\rangle+\left\langle 0\left|\tilde{\mathrm{A}} \mathrm{ex}(-p) \tilde{\mathrm{A}}\left(p_{2}\right) \tilde{\mathrm{A}}\left(p_{3}\right)\right| 0\right\rangle\right]\right\}=0 .
\end{aligned}
$$

Mit Gleichung (101) ergibt sich daraus die Behauptung.

\section{Zusammenfassung und Diskussion}

Ausgehend von verallgemeinerten retardierten Vierpunktfunktionen $r(k)$ bzw. einem trunkierten, vierfachen Vakuumerwartungswert $W^{T}(k)$ haben wir für eine lokale, lorentzkovariante, skalare Feldtheorie unter der Annahme, daß die Massenschale $k^{2}=m^{2}$ isolierter Punkt des EnergieImpuls-Spektrums ist, durch Subtraktion von Produkten von Dreipunktfunktionen neue Funktionen $r^{\mathrm{I}}(k)$ bzw. $W^{\mathrm{II}}(k)$ konstruiert. Diese haben alle Eigenschaften der ursprünglichen Funktionen, außer daß das Spektrum in bestimmten Partialsummen der Impulse erst mit dem Zweiteilchenkontinuum beginnt, d. h., daß die $r^{\mathrm{I}}$-Funktionen Randwerte einer analytischen Funktion $G^{\mathrm{I}}\left(p_{1}, p_{2}, p_{3}, p_{4}\right) p_{j}=k_{j}+i q_{j}$ sind, deren Schnitte in den Variablen $k_{\alpha}+k_{\beta}$ mit $1 \leqq \alpha \neq \beta \leqq 4$ erst bei $4 m^{2}$ beginnen, und daß der Träger von $\widetilde{W}^{\mathrm{II}}\left(k_{1}, k_{2}, k_{3}, k_{4}\right)$ in $k_{1}+k_{2}$ bei der Zweiteilchenschwelle $\left(k_{1}+k_{2}\right)^{2}=4 m^{2}$ beginnt.

Die Tatsache, daß die Lokalität eine in gewisser Weise symmetrische Subtraktion der Produkte der Dreipunktfunktionen erzwingt, legte die Vermutung nahe, daß die $r^{\mathrm{I}}$ - bzw. $W^{\mathrm{II}}$-Funktionen in keiner der Partialsummen $k_{\alpha}+k_{\beta} 1 \leqq \alpha \neq \beta \leqq 4$ auf dem Massenhyperboloid $k^{2}=m^{2}$ eine Singularität haben. Diese Vermutung konnten wir unter der weiteren Voraussetzung der $L$-S-Z-Asymptotenbedingung für $W^{\mathrm{II}}\left(k_{1}, k_{2}, k_{3}, k_{4}\right)$ in $k_{2}+k_{3}$ sowie für $r^{\mathrm{I}}(k)$ in allen diesen Partialsummen zeigen. Da $W^{I I}(k)$ durch die $r^{I}(k)$ eindeutig in linearer Weise darstellbar ist (falls diese existieren), so folgt daraus, daß die Vermutung auch für die restlichen Kombinationen im Falle der Wightman-Funktion richtig ist.

Wir haben also eine Entwicklung der trunkierten WightmanFunktion $W^{T}\left(k_{1}, k_{2}, k_{3}, k_{4}\right)$ gewonnen, in der alle ihre Einteilchensingularitäten in den Impulskombinationen $k_{\alpha}+k_{\beta} 1 \leqq \alpha \neq \beta \leqq 4$ durch die Integralkerne von Produkten von Dreipunktfunktionen explizite gegeben sind. 
Das Konstruktionsverfahren kann prinzipiell für beliebige $n$-Punktfunktionen angewendet werden. Doch erscheint es wegen kombinatorischer Schwierigkeiten ratsamer, in diesem Fall ausgehend von der in Abschnitt IV hergeleiteten Aufspaltung der Wightman-Funktionen auf der Massenschale ein sukzessives Verfahren ohne den Umweg über die verallgemeinerten $r$-Funktionen zu benutzen. Diese Möglichkeit wird zur Zeit noch näher untersucht.

Schließlich haben wir notwendige und hinreichende Bedingungen für die Nichttrivialität von unitären S-Matrizen bzw. einiger ihrer Elemente in Form von Aussagen über das Auftreten von Hauptwertsingularitäten in den Wightman-Funktionen $\widetilde{W} n\left(k_{1}, \ldots, k_{n}\right)$ hergeleitet. Der in diesem Zusammenhang bewiesene Satz VII wurde zuerst von GREenserg und Lichт [45] unter den Annahmen (AI) - (AVI) und der Existenz von $r$-Funktionen sowie von RoBINson [46] unter den Annahmen (AI) bis (AIII) und der in Abschnitt I erwähnten positiven Definitheit bewiesen. Für die hier hergeleiteten Sätze VI-X sind weder die Lorentzkovarianz der Felder (AI) noch die Lokalität (AIII) notwendige Voraussetzungen. Sie gelten in jeder Feldtheorie, in der die Yang-Feldman-Gleichungen gelten, wie z. B. in den nicht kovarianten, quasilokalen Theorien von HAAG [31] und ARAKI [18].

Diese Sätze zeigen, daß in allen quasilokalen, asymptotisch vollständigen Feldtheorien, in denen die Haag-Entwicklung des Feldes nach freien Feldern nur endlich viele von Null verschiedene Entwicklungskoeffizienten hat (wie z. B. die $N$-Quanten-Näherung von GreENBERG [48]), die S-Matrix ein Vielfaches der Einheit ist.

Herrn Professor Dr. H. Lehmann möchte ich für die Anregung zu dieser Arbeit sowie für die Ermunterungen und wertvollen Diskussionen während ihrer Ausführung danken. Frau Dr. U. Völkel und den Herren Dr. H. Joos, Dr. K. Meetz und Dr. H. Satz danke ich für manche kritische Bemerkung und klärende Diskussion.

\section{Anhang}

\section{Integraldarstellungen vom Jost-Lehmann-Dyson-Typ}

Die Matrixelemente $\left\langle 0\left|R\left(z / x_{1}\right) \mathrm{A}\left(x_{2}\right)\right| 0\right\rangle$ und $\left\langle 0\left|\mathrm{~A}\left(x_{2}\right) R\left(z / x_{1}\right)\right| 0\right\rangle$ sollen mit Hilfe von verallgemeinerten Jost-Lehmann-Dyson-Darstellungen präzise definiert werden. $\mathrm{Zu}$ diesem Zweck betrachten wir zunächst die entsprechenden kausalen Kommutatoren:

$$
\left\{\begin{array}{l}
S\left(\left[z, x_{1}\right] x_{2}\right)=:\left\langle 0\left|\left[\mathrm{~A}(z), \mathrm{A}\left(x_{1}\right)\right] \mathrm{A}\left(x_{2}\right)\right| 0\right\rangle \\
S\left(x_{2}\left[z, x_{1}\right]\right)=:\left\langle 0\left|\mathrm{~A}\left(x_{2}\right)\left[\mathrm{A}(z), \mathrm{A}\left(x_{1}\right)\right]\right| 0\right\rangle
\end{array}\right.
$$

bzw. deren Fouriertransformierten

$$
\left.\begin{array}{l}
\tilde{S}\left(\left[p, k_{1}\right] k_{2}\right) \\
\tilde{S}\left(k_{2}\left[p, k_{1}\right]\right)
\end{array}\right\}=\frac{1}{(2 \pi)^{15 / 2}} \int d z d x_{1} d x_{2} e^{i\left(p z+k_{1} x_{1}+k_{2} x_{2}\right)}\left\{\begin{array}{l}
S\left(\left[z ; x_{1}\right] x_{2}\right) \\
S\left(x_{2}\left[z ; x_{1}\right]\right)
\end{array} .\right.
$$


Über die Einteilchensingularitäten in Relativistischen Quantenfeldtheorien 209

Aus der Translationskovarianz folgt:

$$
\left\{\begin{array}{l}
S\left(\left[z, x_{1}\right] x_{2}\right)=S_{1}\left(z-x_{1}, x_{1}-x_{2}\right) \\
S\left(x_{2}\left[z, x_{1}\right]\right)=S_{2}\left(x_{2}-z, z-x_{1}\right)
\end{array}\right.
$$

Definieren wir die Fouriertransformierten $\widetilde{S}_{j}\left(q_{1}, q_{2}\right)$ durch:

$$
\widetilde{S}_{j}\left(q_{1}, q_{2}\right)=\frac{1}{(2 \pi)^{7 / 2}} \int d x_{1} d x_{2} e^{i q x} S_{j}\left(x_{1}, x_{2}\right)
$$

so erhalten wir den Zusammenhang:

$$
\left\{\begin{array}{l}
\tilde{S}\left(\left[p, k_{1}\right] k_{2}\right)=\delta\left(k_{1}+k_{2}+p\right) \tilde{S}_{1}\left(p, k_{1},+p\right) \\
\tilde{S}\left(k_{2}\left[p, k_{1}\right]\right)=\delta\left(k_{1}+k_{2}+p\right) \tilde{S}_{2}\left(k_{2}, p+k_{2}\right) .
\end{array}\right.
$$

Die Distributionen $S_{j}(x, y)$ haben die aus (WI), (WII') und (WIII) folgenden Eigenschaften:

$$
\left\{\begin{array}{l}
\tilde{S}_{j}(p, q)=\tilde{S}_{j}(\Lambda p, \Lambda q) \text { für } \Lambda \in L q \\
\widetilde{S_{1}}(p, q)=0 \text { für } q \notin \overline{V_{+}^{2 m}} ; \tilde{S}_{2}(p, q)=0 \text { für } p \notin \frac{\circ}{V_{+}^{2 m}} \\
S_{1}(x, y)=0 \text { für } x^{2}<0 ; S_{2}(x, y)=0 \text { für } y^{2}<0 .
\end{array}\right.
$$

Es sei $L(q)$ diejenige Lorentztransformation, die den Vektor $q$ drehungsfrei in sein Ruhsystem transformiert [38]:

$$
\begin{gathered}
L(q) q=\left(\sqrt{q^{2}}, 0,0,0\right) \\
L^{-1}(q)_{\mu}^{v}=\frac{1}{\sqrt{q^{2}}}\left(\begin{array}{l}
q^{0} \\
q_{r} \\
q_{t} \sqrt{q^{2}} \delta_{r t}+\frac{q_{r} q_{t}}{\sqrt{q^{2}}+q^{0}}
\end{array}\right) t ; r=1 ; 2 ; 3 .
\end{gathered}
$$

Aus der Lorentzkovarianz folgt dann, daß $\widetilde{S_{j}}(p, q)$ von der folgenden Gestalt sein muß:

$$
\left\{\begin{array}{l}
\tilde{S}_{1}(p, q)=\tilde{\tau}_{1}\left(L(q) p, \sqrt{q^{2}}\right) \\
\tilde{S}_{2}(p, q)=\tilde{\tau}_{2}\left(L(p) q, \sqrt{p^{2}}\right) .
\end{array}\right.
$$

Dabei sind die $\tilde{\tau}_{j}\left(k^{0}, k, t\right)$ temperierte Distributionen über dem Raum $\Im\left(R_{4} *\langle-\infty,+\infty\rangle\right)$, die in $\mathbf{k}$ unter der eigentlichen dreidimensionalen Drehgruppe $0_{+}^{3}$ invariant sind, und deren Träger in der Menge $I_{m}$ mit

enthalten ist:

$$
I_{m}=\{t: t=m \text { oder } 2 m \leqq t<+\infty\}
$$

$$
\begin{gathered}
\tilde{\tau}_{j}\left(k^{0}, R \mathbf{k}, t\right)=\tilde{\tau}_{j}\left(k^{0} ; \mathbf{k} ; t\right) \quad \text { für } \quad R \in 0_{+}^{3} \\
\tilde{\tau}_{j}(k, t)=0 \text { für } t \in I_{m} .
\end{gathered}
$$

Präzise bedeuten die Gleichungen (150) bis (153), daß der Unterraum der temperierten Distributionen aus $\mathfrak{S}^{\prime}\left(R_{8}\right)$ mit den Eigenschaften (147) und der Unterraum der temperierten Distributionen aus $\mathcal{S}^{\prime}\left(R_{4} *\langle-\infty ;+\infty\rangle\right)$ mit den Eigenschaften (152) und (153) topologisch isomorph sind. 
Explizite ist dieser Isomorphismus gegeben durch [39]:

$$
\int d^{4} p d^{4} q \tilde{S}_{1}(p, q) \varphi(p, q)=\int d t d^{4} p \tilde{\tau}_{1}(p, t)(M \varphi)(p, t)
$$

für alle $\varphi(p, q) \in \mathfrak{S}\left(R_{8}\right)$ mit $\varphi(p, q)=0$ für $q \notin \frac{0}{V_{+}^{2 m}}$ und

$$
(M \varphi)(p, t)=: \int d^{4} q \delta\left(q^{2}-t^{2}\right) \varphi\left(L^{-1}(q) p, q\right) .
$$

Führen wir schließlich die partielle Fouriertransformierte

$$
\tau_{j}(x, t)=\frac{1}{(2 \pi)^{3 / 2}} \int d^{4} k \tilde{\tau}_{j}(k, t) e^{-i k x}
$$

ein, so erhalten wir die zu (147) analogen Eigenschaften:

$(\tau \mathrm{I}) \quad \tau_{j}(x, t)=0$ für $x^{2}<0$

( $\tau$ II $) \quad \tau_{j}(k, t)=0$ für $t \notin I_{m}$ oder für $t-\sqrt{m^{2}+\mathbf{k}^{2}}<k^{0}<\sqrt{m^{2}+\mathbf{k}^{2}}$

( $\tau \mathrm{IIII}) \tau_{j}\left(k^{0}, \mathrm{k}, t\right)=\tau_{j}\left(k^{0}, R \mathbf{k}, t\right)$ für $R \in 0_{+}^{3}$.

Die Spektrumseigenschaft $(\tau \mathrm{II})$ ergibt sich leicht folgendermaßen: Durch Einfügen des vollständigen Systems von Zwischenzuständen (64) erhalten wir aus den Darstellungen

$\left\{\begin{array}{l}\tilde{S}_{1}\left(q_{1}, q_{2}\right)=\frac{1}{(2 \pi)^{7 / 2}} \int d^{4} z d^{4} x e^{i\left(q_{1} z+\left(q_{2}-q_{1}\right) x\right)}\langle 0|[\mathrm{~A}(z) ; \mathrm{A}(x)] \mathrm{A}(0)| 0\rangle \\ \tilde{S}_{2}\left(q_{1}, q_{2}\right)=\frac{1}{(2 \pi)^{7 / 2}} \int d^{4} z d^{4} x e^{-i\left(\left(q_{1}-q_{2}\right) z+q_{2} x\right)}\langle 0|\mathrm{~A}(0)[\mathrm{A}(z) ; \mathrm{A}(x)]| 0\rangle\end{array}\right.$

unter Benutzung der Darstellungen (1) und (2) des Translationsoperators:

$$
\begin{aligned}
\tilde{S}_{1}\left(q_{1}, q_{2}\right)= & \operatorname{const} \theta\left(q_{2}\right) \int d \varrho\left(M^{2}\right) \delta\left(q_{2}^{2}-M^{2}\right) \times \\
& \times f_{q_{1}, M}^{(1)}\left(q_{1}-\frac{1}{2} q_{2}\right)\left\langle M, q_{2}|\mathrm{~A}(0)| 0\right\rangle \\
\tilde{S}_{2}\left(q_{1}, q_{2}\right)= & \operatorname{const} \theta\left(q_{1}\right) \int d \varrho\left(M^{2}\right) \delta\left(q_{1}^{2}-M^{2}\right) \times \\
& \times f_{q_{2}, M}^{(2)}\left(q_{2}-\frac{1}{2} q_{1}\right)\left\langle 0|\mathrm{~A}(0)| q_{1} ; M\right\rangle
\end{aligned}
$$

mit

$$
\left\{\begin{array}{l}
f_{p, M}^{(j)}(q)=\int d^{4} x e^{i q x} f_{p ; M}^{(j)}(x) \\
f_{p, M}^{(2)}(x)=:\left\langle M ; p\left|\left[\mathrm{~A}\left(\frac{x}{2}\right) ; \mathrm{A}\left(-\frac{x}{2}\right)\right]\right| 0\right\rangle \\
f_{p, M}^{(1)}(x)=:\left\langle 0\left|\left[\mathrm{~A}\left(\frac{x}{2}\right) ; \mathrm{A}\left(-\frac{x}{2}\right)\right]\right| p ; M\right\rangle .
\end{array}\right.
$$

Aus Gleichung (9) ergibt sich dann mit der Lorentzkovarianz:

$$
\begin{aligned}
\tilde{\tau}_{1}\left(L(q) p, \sqrt{q^{2}}\right)= & \text { const } \int d \varrho\left(M^{2}\right) \delta\left(q^{2}-M^{2}\right) \theta\left(q^{0}\right) \times \\
& \times \tilde{f}_{L(q) q, M}^{(1)}\left(L(q)\left(p-\frac{1}{2} q\right)\right)\langle M, L(q) q|\mathrm{~A}(0)| 0\rangle \\
\tilde{\tau}_{2}\left(L(q) p, \sqrt{q^{2}}\right)= & \text { const } \int d \varrho\left(M^{2}\right) \delta\left(q^{2}-M^{2}\right) \theta(q) \times \\
& \times f_{L(q) q, M}^{(2)}\left(L(q)\left(p-\frac{1}{2} q\right)\right)\langle 0|\mathrm{~A}(0)| L(q) q, M\rangle .
\end{aligned}
$$


Mit den bekannten Trägereigenschaften von $\tilde{f}_{p, M}^{j}(q)[40]$ :

$$
\begin{gathered}
\tilde{f}_{L(q) q, M}^{(j)}(L(q) p) \neq 0 \quad \text { für } \quad(L(q) p)^{0} \geqq-\frac{\sqrt{q^{2}}}{2}+\sqrt{m^{2}+\mathbf{P}_{q}^{2}} \\
\text { oder für }(L(q) p)^{0} \leqq \frac{\sqrt{q^{2}}}{2}-\sqrt{m^{2}+\mathbf{P}_{q}^{2}} \\
\text { mit } P_{q}=: L(q) p
\end{gathered}
$$

folgt dann die Behauptung ( $\tau$ II).

Mit genau den gleichen Überlegungen wie bei der Herleitung der gewöhnlichen Jost-Lehmann-Dyson-Darstellung [40], [41] (Erweiterung von $\tilde{\tau}_{j}(k, t) \mathrm{zu}$ einer rotationssymmetrischen, temperierten Lösung der sechsdimensionalen, hyperbolischen Wellengleichung und Lösung des zugehörigen Cauchy-Problems unter Benutzung des Doppelkegeltheorems) beweist man den Satz:

Satz XII. Eine temperierte Distribution hat dann und nur dann die Eigenschaften $(\tau \mathrm{I})-(\tau \mathrm{III})$, wenn sie in der folgenden Form darstellbar ist:

$$
\begin{aligned}
\tilde{\tau}_{j}(p, t) & =\int d^{3} \mathbf{u} d s \varepsilon\left(p^{0}-\frac{t}{2}\right) \delta\left(\left(p^{0}-\frac{t}{2}\right)^{2}-(\mathbf{p}-\mathbf{u})^{2}-s\right) \times \\
& \times\left[\Phi_{j}^{1}(\mathbf{u}, s, t)+\left(2 p^{0}-t\right) \Phi_{j}^{2}(\mathbf{u}, s, t)\right] .
\end{aligned}
$$

Dabei sind die Spektralfunktionen $\Phi_{j}^{i}(\mathbf{u}, s, t) \quad 0_{+}^{3}$-invariante temperierte Distributionen, deren Träger für festes $t \in I_{m}$ in der Menge $\Gamma$ mit

$$
\Gamma=:\left\{(\mathbf{u} ; s):|\mathbf{u}| \leqq \frac{t}{2} ; s \geqq \max \left[0 ; m-\sqrt{\frac{t^{2}}{4}-|\mathbf{u}|^{2}}\right]\right\}
$$

enthalten ist. Umgekehrt sind die Spektralfunktionen $\Phi_{j}^{i}$ für vorgegebenes $\tilde{\tau}_{j}(p, t)$ eindeutig gegeben durch:

$$
\begin{aligned}
\Phi_{j}^{1}(\mathbf{u}, s, t) & =\frac{1}{2 \pi} \frac{d}{d s}\left[\theta(s) \frac{\partial}{\partial u^{0}} G_{j}\left(u_{1}^{0}, \mathbf{u}, s, t\right)_{u^{0}=\frac{t}{2}}\right] \\
\Phi_{j}^{2}(\mathbf{u}, s, t) & =\frac{1}{2 \pi} \frac{d^{2}}{d s^{2}}\left[\theta(s) G_{j}\left(\frac{t}{2}, \mathbf{u}, s, t\right)\right] \\
G_{j}(u, s, t) & =\frac{1}{(2 \pi)^{5 / 2}} \int d^{4} x l^{i u x} J_{0}\left(\sqrt{s x^{2}}\right) \tilde{\tau}_{j}(x, t) \\
& =\frac{1}{\pi^{2}} P \int d^{4} p \frac{\tilde{\tau}_{j}(p, t)}{\left[(p-u)^{2}-s\right]^{2}} .
\end{aligned}
$$

Ausgehend von der Darstellung (162) kann man nun mit dem üblichen Konstruktionsverfahren [43] zu einem bis auf Polynome in $k^{0}$ wohl definierten Ausdruck für den retardierten bzw. avancierten Kommutator $\tau_{r}^{(j)}(x, t)={ }^{\prime \prime} \theta\left( \pm x^{0}\right) \tau_{j}(x, t)^{\prime \prime}$ gelangen. Dieses Konstruktionsverfahren sei hier nur angedeutet und für die Einzelheiten auf den Artikel von OMnes [43] verwiesen.

Zunächst ist $\tau_{j}(x, t)$ für festes $t$ im Ursprung $x^{0}=0$ von endlicher Ordnung, d. h. es existiert eine ganze Zahl $n$ der Art, daß $\left(x^{0}\right)^{2 n} \tau_{j}\left(x^{0}, x, t\right)$ 
dort regulär ist, und daher $\theta\left( \pm x^{0}\right)\left(x^{0}\right)^{2 n} \tau_{j}(x, t)$ wohl definiert ist. Da die Distributionen

bzw.

$$
g_{j}(x, t)=\left(x^{0}\right)^{2 n} \tau_{j}(x, t)
$$

$$
\tilde{g}_{j}(q, t)=\left(-i \frac{\partial}{\partial q^{0}}\right)^{2 n} \tilde{\tau}_{j}(q, t)
$$

dieselben Träger- und Invarianzeigenschaften wie $\tau_{j}(x, t)$ bzw. $\tilde{\tau}_{j}(k, t)$ haben, und die Faltung

$$
\left(-i \frac{\partial}{\partial q^{0}}\right)^{2 n} \underset{a}{\tilde{\tau}_{r}^{(j)}}(q, t)=\left(\frac{1}{q^{0}-q^{0} \pm i \varepsilon} \otimes \tilde{g}(q, t)\right)
$$

existiert, so erhält man nach einigen Rechnungen aus Gleichung (162) unter Ausnutzung der Tatsache, daß der Träger von $\Phi_{j}^{i}(u, s, t)$ in $|\mathrm{u}|$ beschränkt ist:

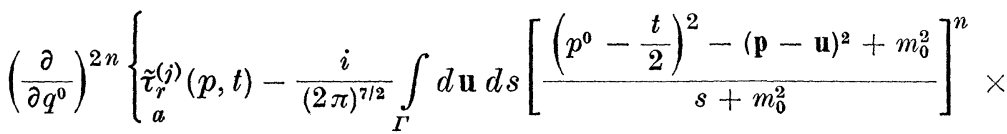

$$
\begin{aligned}
& \left.\frac{\Phi_{j}^{1}(\mathbf{u}, s, t)+\left(2 p^{0}-t\right) \Phi_{j}^{2}(\mathbf{u}, s, t)}{\left(p^{0}-\frac{t}{2} \pm i \gamma\right)^{2}-(\mathbf{p}-\mathbf{u})^{2}-s}\right\}=0
\end{aligned}
$$

oder

$$
\begin{aligned}
& \underset{a}{\tilde{\tau}_{r}^{(j)}}(p, t)=+\frac{i}{(2 \pi)^{2 / 2}} \int_{\Gamma} d \mathbf{u} d s \frac{\left[(p-u)^{2}+m_{0}^{2}\right]^{n}}{\left(s+m_{0}^{2}\right)^{n}\left[(p-u \pm i \gamma)^{2}-s\right]} \times \\
& \times\left[\Phi_{j}^{1}(\mathbf{u}, s, t)+\left(2 p^{0}-u^{0}\right) \Phi_{j}^{2}(\mathbf{u}, s, t)\right]_{u^{0}=\frac{t}{2}}+\sum_{j=0}^{2 n-1} c_{j}\left(p^{0}\right)^{j}
\end{aligned}
$$

Da in den Gleichungen (91) bzw. (100) das in (168) auftretende Polynom infolge des Klein-Gordon-Operators $\left(p^{2}-m^{2}\right)$ auf der Massenschale keinen Beitrag liefert, so können wir ohne Einschränkung der Allgemeinheit die dort auftretenden retardierten Kommutatoren durch die folgenden Darstellungen definieren:

$$
\begin{aligned}
& \left\langle 0\left|\widetilde{R}\left(p \mid k_{1}\right) \tilde{\mathrm{A}}\left(k_{2}\right)\right| 0\right\rangle=: \delta\left(k_{1}+k_{2}+p\right) \tilde{\tau}_{r}^{(1)}\left(L\left(k_{1}+p\right) p, \sqrt{\left(k_{1}+p\right)^{2}}\right) \\
& \left\langle 0\left|\tilde{\mathrm{A}}\left(k_{2}\right) \widetilde{R}\left(p / k_{1}\right)\right| 0\right\rangle=: \delta\left(k_{1}+k_{2}+p\right) \tilde{\tau}_{r}^{(2)}\left(L\left(k_{2}\right)\left(k_{2}+p\right), \sqrt{k_{2}^{2}}\right)
\end{aligned}
$$

mit

$$
\begin{gathered}
\tau_{r}^{(j)}(p, t)=\frac{i}{2 \pi^{7 / 2}} \int_{\Gamma} d \mathbf{u} d s \frac{\left[(p-u)^{2}+m_{0}^{2}\right]^{n}}{\left[s+m_{0}^{2}\right]^{n}\left[(p-u+i \gamma)^{2}-s\right]} \times \\
u^{0}=\frac{t}{2} \\
\times\left[\Phi_{j}^{1}(\mathbf{u}, s, t)+2\left(p^{0}, u^{0}\right) \Phi_{j}^{2}(\mathbf{u}, s, t)\right] \quad m_{0}^{2}>0 ; \gamma_{0}=(\gamma, \mathbf{0}) ; \gamma>0
\end{gathered}
$$

Bei vorgegebenen Kommutatoren $\tilde{\tau}_{1}\left(p, \sqrt{q^{2}}\right)=\widetilde{S}_{1}(p, L(q) q)$ und $\tilde{\tau}_{2}\left(p, \sqrt{q^{2}}\right)$ 
$={\widetilde{S_{2}}}_{2}(L(q) q, p)$ sind die Spektralfunktionen $\Phi_{j}^{i}\left(\mathbf{u}, s, \sqrt{q^{2}}\right)$ durch die Gleichungen (164) und (165) eindeutig gegeben.

Da die Matrixelemente (169) und (170) bis auf Polynome im Impulsraum, deren Beitrag in den Gleichungen (95) - (98) sowie in Gleichung (100) auf der Massenschale verschwinden würde, eindeutig definiert sind, erhalten wir den Satz:

Satz XIII. Für gegebene temperierte Distributionen $W\left(x_{1}, x_{2}, x_{3}\right)$ und $W^{T}\left(x_{1}, x_{2}, x_{3}, x_{4}\right)$ mit den Eigenschaften (WI), (WII') und (WIII) erfüllen die durch die Gleichungen (164), (165), (169)-(170), (91) und (100) definierten temperierten Distributionen $W_{\mathfrak{R}}^{\mathrm{II}}\left(x_{1}, x_{2}, x_{3}, x_{4}\right)$ die Bedingungen (WI), (WII') und (WIII) für jeden Kern $\Re(x)$ mit den Eigenschaften $(\Re)$. Die daraus konstruierte temperierte Distribution

$$
\bar{W}_{\mathfrak{R}}\left(x_{1}, x_{2}, x_{3}, x_{4}\right)=\prod_{r=1}^{4} K_{x_{r}}^{m} W_{\mathfrak{R}}^{\mathrm{II}}\left(x_{1}, x_{2}, x_{3}, x_{4}\right)
$$

hat neben den Eigenschaften (WI) und (WIII) die Trägereigenschaft ( $\overline{\mathrm{W}} \mathrm{II})$.

Die Lorentz-Kovarianz ist in dem obigen Satz durch den topologischen Isomorphismus zwischen dem Unterraum der temperierten (Lorentz-kovarianten) Distribution mit den Eigenschaften (147) und dem Unterraum der temperierten $\left(0_{3}\right.$-kovarianten) Distribution mit den Eigenschaften (152) und (153) gesichert.

\section{Literatur}

[1] Schwartz, L.: Theorie des distributions I, II. Paris: Hermann 1957 und 1959.

[2] Gelfand, I., u. G. E. Schilow: Verallgemeinerte Funktionen I-IV. Berlin: Deutscher Verlag der Wissenschaften 1960.

[3] Garding, L., u. J. L. Lions: Nuovo cimento, Suppl. XIV, 9 (1959).

[4] Streater, R. F., and A. S. Wightman: PCT, Spin and Statistics and all that. New York: Benjamin 1964.

[5] Wrghtman, A. S.: Phys. Rev. 101, 860 (1956).

[6] Schwartz, L.: Medd. Lunds. Univ. Mat. Sem. Suppl. 1952, S. 196.

[7] Hall, D., og A. S. Wightman: Kgl. Danske Videnskab. Selskab. Mat.-fys. Medd. 31, No. 5 (1957).

[8] Araki, H.: Progr. Theor. Phys. (Kyoto) Suppl. 18, 83 (1961).

[9] Jost, R.: Helv. Phys. Acta 32, 409 (1957).

[10] Tomozawa, Y.: J. Math. Phys. 4, 1241 (1963).

[11] HAAG, R.: Phys. Rev. 112, 669 (1958).

[12] Araki, H.: J. Math. Phys. 2, 163 (1961).

[13] Borchers, H. J.: Nuovo cimento 24, 214 (1962).

[14] - Nuovo cimento 33, 1600 (1964).

[15] Ruelle, D.: Helv. Phys. Acta 35, 147 (1962).

[16] Hepp, K., u. R. Jost: Helv. Phys. Acta 35, 34 (1962).

[17] Araki, H., K. Hepp u. D. Ruelle: Helv. Phys. Acta 35, 164 (1962).

[18] - Vorlesungen über relativistische Quantenfeldtheorie. E.T.H. Zürich (1962).

[19] Hepp, K.: Commun. Math. Phys. 1, 95 (1965). 
[20] Lemmann, H., K. Symanzik u. W. Zimmermann: Nuovo cimento 1, 205 (1955).

[21] - Nuovo cimento 6, 319 (1957).

[22] Symanzik, K.: J. Math. Phys. 1, 249 (1960).

[23] - Lectures in Theoretical Physics. Vol. III. Boulder 1960. New York: Interscience Publishers 1961.

[24] Zimmermann, W.: Nuovo cimento 13, 503 (1959).

[25] - Nuovo cimento 16, 690 (1960).

[26] Steinmann, O.: Helv. Phys. Acta 33, 257 u. 347 (1960).

[27] RUelle, D.: Nuovo cimento 19, 356 (1961).

[28] Bros, J., H. Epstein u. V. Glaser: Nuovo cimento 31, 1265 (1964).

[29] Araki, H., u. N. Burgoyne: Nuovo cimento 18, 342 (1960).

[30] Jost, R.: Helv. Phys. Acta 31, 263 (1958).

[31] HaAG, R., and B. Schroer: J. Math. Phys. 3, 248 (1962).

[32] Steinmann, O.: Helv. Phys. Acta 36, 90 (1963).

[33] Stora, R.: Preprint. Sacley, 1965.

[34] NishiJima, K.: Progr. Theor. Phys. (Kyoto) 17, 765 (1957).

[35] Glaser, V., H. Lemmann u. W. Zimmermann: Nuovo cimento 6, 1122 (1957).

[36] Epstein, H.: J. Math. Phys. 1, 524 (1960).

[37] Schweber, S. S.: An Introduction to Relativistic Quantum Field Theory. New York: Row, Peterson 1961.

[38] Joos, H.: Fortschr. Physik 10, 65 (1962).

[39] Hepp, K.: Helv. Phys. Acta 37, 639 (1964).

[40] Jost, R., u. H. LehmanN: Nuovo cimento 5, 1598 (1957).

[41] Dyson, F. J.: Phys. Rev. 110, 1460 (1958).

[42] Wightman, A. S.: In: Dispersion relations and elementary particles. Paris: Hermann 1960.

[43] Omnes, R.: In : Dispersion relations and elementary particles. Paris: Hermann 1960.

[44] HaAg, R., u. D. W. Robinson: Seminarvortrag von R. HAAG Hamburg $1964 / 65$.

[45] Greenberg, O. W., and A. L. Licht: J. Math. Phys. 4, 613 (1963).

[46] Robinson, D. W.: Commun. math. Phys. 1, 57 (1965).

[47] Schroer, B.: J. Math. Phys. 5, 1361 (1964).

[48] Greenberg, O. W.: Phys. Rev. 139B, 1038 (1965). 